JOURNAL OF MECHANICS IN MEDICINE AND BIOLOGY

(ARTICLE ID: JMMB D 1600086 )

Print ISSN: 0219-5194

Online ISSN: 1793-6810

IMPACT FACTOR $=0.797$

PUBLISHER: WORLD SCIENTIFIC (SINGAPORE)

ACCEPTED AUGUST 15 ${ }^{\text {TH }} 2016$

\title{
Swimming dynamics of a micro-organism in a couple stress fluid: $a$ rheological model of embryological hydrodynamic propulsion *
}

\author{
N. Ali ${ }^{\mathrm{a}}$, M. Sajid ${ }^{\mathrm{b}}$, Z. Abbas ${ }^{\mathrm{c}}$ and O. Anwar Bég ${ }^{\mathrm{\wedge}}$ \\ ${ }^{a}$ Department of Mathematics \& Statistics, International Islamic University, Islamabad, Pakistan \\ ${ }^{b}$ Theoretical Plasma Physics Division, PINSTECH, P.O. Nilore, Islamabad 44000, Pakistan \\ ${ }^{c}$ Department of Mathematics, The Islamia University of Bahawalpur,Bahawalpur 63100, Pakistan \\ ${ }^{d}$ Fluid Mechanics and Propulsion, Mechanical and Aeronautical Engineering, School of Computing, \\ Science and Engineering, UG17, Newton Building, University of Salford, M5 4WT, UK.
}

\section{^Corresponding author-email:O.A.Beg@salford.ac.uk; gortoab@gmail.com}

\begin{abstract}
Mathematical simulations of embryological fluid dynamics are fundamental to improving clinical understanding of the intricate mechanisms underlying sperm locomotion. The strongly rheological nature of reproductive fluids has been established for a number of decades. Complimentary to clinical studies, mathematical models of reproductive hydrodynamics provide a deeper understanding of the intricate mechanisms involved in spermatozoa locomotion which can be of immense benefit in clarifying fertilization processes. Although numerous non-Newtonian studies of spermatozoa swimming dynamics in non-Newtonian media have been communicated, very few have addressed the micro-structural characteristics of embryological media. This family of micro-continuum models include Eringen's micro-stretch theory, Eringen's microfluid and micropolar constructs and V.K. Stokes' couple-stress fluid model, all developed in the 1960s. In the present paper we implement the last of these models to examine the problem of micro-organism (spermatozoa) swimming at low Reynolds number in a homogenous embryological fluid medium with couple stress effects. The micro-organism is modeled as with Taylor's classical approach, as an infinite flexible sheet on whose surface waves of lateral displacement are propagated. The swimming speed of the sheet and rate of work done by it are determined as function of the parameters of orbit and the couple stress fluid parameter $(\alpha)$. The perturbation solutions are validated with a Nakamura finite difference algorithm. The perturbation solutions reveal that the normal beat pattern is effective for both couple stress and Newtonian fluids only when the amplitude of stretching wave is small. The swimming speed is observed to decrease with couple stress fluid parameter tending to its Newtonian limit as alpha tends to infinity. However the rate of work done by the sheet decreases with $\alpha$ and approaches asymptotically to its Newtonian value. The present solutions also provide a good benchmark for more advanced numerical simulations of micro-organism swimming in couple-stress rheological biofluids.
\end{abstract}

Keywords: Spermatozoa micro-organism, swimming sheet, couple stress non-Newtonian fluid, embryological fluid dynamics, beat pattern, amplitude, energy dissipation, propulsive velocity, perturbation, Nakamura method.

* Dedicated respectfully to Professor T.K. Hung, Bioengineering, University of Pittsburgh, USA, in recognition of his many contributions to engineering biofluid dynamics on the occasion of his $80^{\text {th }}$ birthday (2016). 


\section{INTRODUCTION}

The dynamics of swimming micro-organisms has attracted the attention of many researchers due to extensive applications in medical engineering, biology and ecology. It has been observed that microorganisms like spermatozoa (with characteristic dimensions of $5 \times 10^{-3}$ length and $10^{-5} \mathrm{~cm}$ tail diameter) propel themselves by propagating waves of lateral displacement down a thin tail or flagellum [1]. The swimming strategies used by micro-organisms and larger animals such as fish are similar in many respects (despite the difference in scale) in the sense that in both the propulsion is achieved by utilizing waves of lateral displacement. In aquatic species (e.g. fish) these waves travel down the creature's body [2] whereas in micro-organisms they propagate down a thin tail or flagellum. However, large swimmers make use of inertia of the surrounding fluid whereas micro-organisms use viscous forces for propulsion since in the case of micro-organisms stresses due to viscosity are thousands of times in excess as those due to inertia. The swimming sheet model was proposed by the celebrated English applied mathematician, G.I. Taylor in a seminal and ground-breaking mathematical study over six decades ago [3], encouraged by his interaction with zoologists. Taylor used this approach to discuss the self-propulsion of an infinite waving sheet in a fluid with negligible inertia. In [2] Taylor also discussed the synchronization of swimming micro-organisms by analyzing the field of flow between two infinite and parallel waving sheets. In another paper [3] Taylor modeled the micro-organism as an infinite cylindrical filament. The model of micro-swimmer presented in [2] served as a base for further developments in the field of low Reynolds number locomotion. Shortly after Taylor's work Hancock [4] showed that the motion of a small cylinder perpendicular to its axis is twice as effective in producing "general motion" as moving the cylinder parallel to itself. Later a remarkable attempt was presented by Gray and Hancock [5] which was successfully applied to obtain the velocity of sea urchin spermatozoa. Further studies related to flagella motion can be found in refs. [6-8]. Taylor's results were extended to larger amplitudes of the sheet's motion by Drummond [9]. Inertial effects in the analysis were included by Reynolds [10]. His results indicate that swimming speed decreases with Reynolds number i.e. propulsive efficiency is a maximum at very low Reynolds numbers. Tuck [11] modified the results of Reynolds by correctly incorporating the convective acceleration term in the Navier-Stokes equations. The problem of a swimming sheet in the limit of high Reynolds number has been addressed by Childress [12]. Blake [13] argued that the model of infinite waving sheet is appropriate for elongated and flat micro-organisms such as Paramecium or Opalina. He thus modeled collaboratively beating cilia as an infinite impenetrable surface assuming that the cilia are sufficiently packed together. Propulsion of cilia and flagella was also discussed by Brennen [14] and Brennen and Winet [15]. 
Felderhof [16] showed that peristaltic pumping with two waving walls is closely related to Taylor's analysis [2] of hydrodynamic interaction of two swimming sheets in infinite space. A relationship between peristaltic pumping and two waving parallel surfaces is also discussed by Jaffrin and Shapiro [17] and Fauci and Dillon [18]. In a recent paper Pak and Lauga [19] have discussed the transient swimming of a waving sheet immersed in a viscous fluid.

It is observed that micro-organisms often swim in narrow passages. This is certainly familiar in the realm of embryological transport. Therefore it seems that the presence of solid boundaries (whether rigid or deformable) may have some impact on the locomotion of micro-organisms. This observation has motivated some interesting efforts in reproductive fluid mechanics modelling. Reynolds [10] has simulated the motion of spermatozoa through the cervix as a self-propelling infinite oscillating sheet between two rigid walls and obtained the solution under the assumption that the amplitude of the waving sheet is small compared to the gap between the walls. The model introduced by Reynolds was further examined by Shack and Lardner [20] for the case when the wavelength of the motion of the swiming sheet is large compared to the channel spacing. The assumption of rigid passive walls in the Reynolds model [10] was removed by Smelser et al. [21] in favor of vibrating active walls. Katz [22] performed the analysis for the case when micro-organisms swim either parallel to a single rigid plane wall, or along a channel formed by two such walls. The effects of dynamical interaction of the micelles with the swimming sperm and a more viscous peripheral layer due to concentration and alignment of micelles near the wall were elaborated by Shukla et al. [23]. In a further investigation, Shukla et al. [24] modeled the motion of spermatozoa in the cervical canal by considering the transverse waves along its tail and the transverse and longitudinal motions of the cervical wall, observing that the appropriate motion of cervical canal may restrict the sperm in moving towards the oviduct.

Extensive clinical research over the past decades, has established that the majority of biological fluids including cervical and respiratory mucus for instance, are strongly non-Newtonian in nature. Therefore, classical Navier-Stokes theory (based on the Newtonian law of viscosity) is inadequate for simulating the hydrodynamics of such fluids. To overcome this shortcoming, Chaudhury [25] extended the Taylor's swimming sheet problem to viscoelastic second order fluids. Sinha et al. [26] analyzed selfpropulsion of spermatozoa through the cervical mucus by modeling the mucus as a micropolar fluid ( a simplification of the more general micro-morphic fluid theory) and the cervical canal as a channel composed of rigid walls. The analysis in [26] was extended to take into account the sinusoidal vibrations of the flexible walls of the channel by Philip and Chandra [27]. Lauga [28] further emphasized the need for employing robust non-linear non-Newtonian models for swimming in a 
viscoelastic medium such as mucus. His calculations showed that viscoelastic effects slow down the speed of the swimming sheet for a prescribed beating pattern on the sheet. Using a two-dimensional infinite sheet model, Elfring et al. [29] presented an analytical treatment of synchronization in a viscoelastic Oldroyd-B fluid. Fu et al. [30] employed an infinitely long cylinder geometric model with arbitrary beating motion in an Oldroyd-B fluid to elucidate how swimming velocities are affected in nonlinear viscoelastic fluids, revealing that nonlinear viscoelastic corrections decrease the swimming speed relative to the Newtonian case. The same model with a prescribed beating pattern on the cylinder in a Maxwell viscoelastic fluid was also analyzed by $\mathrm{Fu}$ et al. [31], identifying that viscoelasticity tends to decrease the swimming speed. More recently Teran et al. [32] showed that viscoleastic response can increase the speed and efficiency of free swimmers. Balmforth et al. [33] exploited the lubrication approximation to model the motion of sperm near a rigid wall immersed in a complex fluid, combining a simple generalized Newtonian fluid model and a Bingham fluid model. Many other excellent studies of propulsion in non-Newtonian fluids at low Reynolds number have been communicated by Lauga and co-workers [34]-[37] which consider viscoplastic, highly viscous and elastic-viscous biological media as the propagating environments.

Inspection of the medical scientific and engineering literature indicates that the swimming problem has thusfar not been analyzed for the case of a couple stress fluid. In the category of non-Newtonian fluids, couple stress fluids exhibit distinct micro-structural features such as polar effects in addition to possessing large viscosity. A comprehensive theory in this regard is that developed by Stokes [38]. This theory is a generalization of classical theory which allows for polar effects such as the presence of couple stresses and body couples. The main effect of couple stresses is to introduce a size-dependent effect that is not present in the classical viscous theories. In the latter the stress tensor is symmetric as a result of assuming there is no rotational interaction among particles. However, this cannot be true for the cases of fluid flow with suspended particles and thus the need of couple stress theory arises. In fact the microrotation of freely suspended particles give rise to an antisymmetric stress, known as couple stress. Couple stress theory is found quite useful in description of various types of lubricants, blood, and also polymeric suspensions [39]. It is also pertinent to mention that couple stress fluid theory is substantially simpler than other micro-structural theories, pre-eminent among which is the Eringen micropolar fluid model. Both models and infact more complex models are reviewed lucidly by the premier researcher in the field, Eringen [39]. Couple stress fluids do not have micro-structure at the kinematic level and this allows the kinematics of such fluids to be entirely described using the velocity field. The need for a separate spin momentum (micro-rotation) balance is therefore not required, as 
with micropolar fluids. Effectively in Stokes [38] couple stress model, micro-structural effects are considered as a consequence of the action of a deforming body on its neighborhood. Application of the couple stress model to physiological and medical engineering problems has been quite diverse. Studies include double-diffusive transport as studied by Srinivasacharya and Kaladher [40] and peristaltic transport as investigated by Srivastava [41], Shehawey and Mekheimer [42], Ali et al. [43] and Tripathi and Bég [44]. Further biomedical topics in which couple stress theory has been utilized include blood flow in the microcirculation [45], centrifugal blood purification devices [46], pulsatile systolic hemodynamics [47] and synovial tribology [48]. According to [49] it is more likely that couple stresses arise in fluids with very large molecules. Further it is observed that cervical mucus is a suspension of macro-molecules with a high molecular weight $[\mathbf{5 0 , 5 1}]$ and in light of this, couple stress theory may be applied to analyze the swimming of spermatozoa through cervical mucus. So far only a few mathematical studies- see references [24], [26] and [27] - have been communicated concerning swimming of microscopic organism in micro-continuum fluids. However, the micro-continuum fluid model chosen in these studies is Eringen's micropolar fluid model [39], not Stokes simpler couple stress model.

Motivated by the above facts, the aim of the present communication is to analyze the effects of couple stresses on the locomotion of spermatozoa micro-organisms in cervical hydrodynamics. The microorganism is modeled as an infinite flexible waving sheet. The paper is structured as follows: Section 2 presents the governing equations along with the boundary conditions. The solution of the problem is presented in section 3. Section 4 comprises numerical validation. Section 5 entails graphical presentation and discussion of results. The paper ends with some concluding remarks in section 6.

\section{GOVERNING EQUATIONS}

Let us consider an infinite flexible sheet of infinitesimal thickness. The sheet is swimming steadily in a viscous incompressible fluid with couple stresses. In a frame moving at the swimming speed of the sheet (which is yet unknown), the position of the material points, $\left(x_{s}, y_{s}\right)$ of the sheet is given by the following relations:

$$
\begin{aligned}
x_{s} & =x+a \cos (k x-\omega t-\phi) \\
& =x+\beta^{*} \cos (k x-\omega t)+\gamma^{*} \sin (k x-\omega t), \\
y_{s} & =b \sin (k x-\omega t) .
\end{aligned}
$$

In eqns. (1) and (2) $\beta^{*}=a \cos \phi$ and $\gamma^{*}=a \sin \phi$. These equations represent tangential movements of 
the sheet in addition to the progressive wave. The case of a simple progressive wave can be achieved by substituting $a=0$ (Fig. 1). The flow is subject to the constraint of incompressibility given by:

$$
v_{k, k}=0,
$$

For a couple stress fluid of viscosity, $\mu$, and density, $\rho$, the constitutive equations and equation of motion in the absence of body forces and body moments, in tensorial form, are [38]:

$$
\begin{gathered}
T_{j i, j}=\rho \frac{d v_{i}}{d t}, \\
e_{i j k} T_{j k}^{A}+M_{j i, j}=0 \\
\tau_{i j}=-p \delta_{i j}+2 \mu d_{i j}, \\
\mu_{i j}=4 \eta \omega_{j, i}+\eta^{\prime} \omega_{i, j},
\end{gathered}
$$

where $v_{i}$ is the velocity vector, $\tau_{j k}$ and $T_{j k}^{A}$ are the symmetric and anti-symmetric parts of the stress tensor $T_{j k}$, respectively, $M_{i j}$ is the couple stress tensor, $\mu_{i j}$ is the deviatoric part of $M_{i j}$, $\omega_{i}=1 / 2 e_{i r s} u_{s, r}$ is the angular velocity vector, $d_{i j}$ is the symmetric part of the velocity gradient, $\eta$ and $\eta^{\prime}$ are constants associated with the couple stress, $p$ is the pressure and other terms have their usual meaning from the tensor analysis. Stokes' micro-continuum "couple stress fluid" theory is a generalization of the classical theory of fluids where polar effects are captured including couple stresses and body couples. It is therefore a more realistic constitutive model in which the couple stress effects are considered as a consequence of the action of a deforming body on its neighbourhood. The key characteristic of couple stresses is to introduce a length-dependent effect via extra derivatives, which are linear and therefore more easily simulated than micropolar fluid theory. Couple stress theory is therefore much simpler than other nonlinear micro-stuctural non-Newtonian models, yet representative of the physics of many important thermal process fluids. The main effect of couple stresses is to model "micro-structure" as a size-dependent effect that is not present in the classical viscous theories i.e. parcels of fluid can sustain couple stresses. The effect of very small microstructure in a fluid can be felt if the characteristic geometric dimension of the problem considered is of the same order of magnitude as the size of the microstructure. Classical continuum mechanics neglects the size effect of material particles within the continua. This is consistent with ignoring the rotational interaction among particles, which results in symmetry of the force-stress tensor. Couple stresses are incorporated by assuming that the mechanical action of one part of a body across a surface is equivalent to a force and moment distribution, unlike with Navier-Stokes fluids (non-polar fluids) 
where the moment is ignored. This approach is more elegant than other non-Newtonian models e.g. viscoplastic, viscoelastic, thixotropic etc, which merely modify the shear stress-strain characteristics of Newtonian fluids via extra stress tensors. Such non-polar models cannot simulate micro-structural or rotational (micro-vorticity) motions which are significant in fluids with suspensions and complex micro-structure. Couple stress fluids therefore are a more sophisticated approach to analyzing real physiological fluids for which other rheological models (e.g. viscoelastic/plastic) neglect many important features. An excellent elaboration of the continuum physics of couple stress fluids is available in Cowin [52]. Since the velocity field in the current problem, is two-dimensional therefore we can take $v_{1}=u(x, y, t)$ and $v_{2}=v(x, y, t)$. Moreover a dimensional stream function may be defined, by the relations $u=\partial \psi / \partial y$ and $v=-\partial \psi / \partial x$, and, under the assumption of negligible inertia Eqn. (4) can be written in component form as:

$$
\begin{aligned}
& p_{x}-\mu \nabla^{2} \psi_{y}+\eta \nabla^{4} \psi_{y}=0, \\
& p_{y}+\mu \nabla^{2} \psi_{x}-\eta \nabla^{4} \psi_{x}=0,
\end{aligned}
$$

where subscripts denote partial differentiation with respect to $x$ or $y$. It should be noted that Eqn. (3) is satisfied identically by using the definition of stream function. The boundary conditions for velocity at the sheet surface read:

$$
\begin{aligned}
& \left.\psi_{y}\right|_{\left(x_{s}, y_{s}\right)}=\beta^{*} \omega \sin (k x-\omega t)-\gamma^{*} \omega \cos (k x-\omega t), \\
& \left.\psi_{x}\right|_{\left(x_{s}, y_{s}\right)}=b \omega \cos (k x-\omega t), \\
& \left.b k \cos (k x-\omega t)\left(\psi_{x x x}+\psi_{x y y}\right)\right|_{\left(x_{s}, y_{s}\right)}-\left.\left(\psi_{x x y}+\psi_{y y y}\right)\right|_{\left(x_{s}, y_{s}\right)}=0 .
\end{aligned}
$$

The first two boundary conditions represent the no-slip at the sheet surface and for the third condition we have assumed that couple stress tensor must vanish at the sheet surface. The assumption of zero angular velocity at the sheet implies the vanishing of couple stresses at the sheet and hence traction due to couple stresses must also be zero there. The condition (12) is precisely the mathematical expression of the statement that traction due to couple stresses vanish at the sheet surface and has been rigorously verified and successfully deployed by several authors in the literature [e.g. 38, 44-48]. Eliminating pressure from Eqns. (8) and (9) results in the following equation in terms of $\psi$ :

$$
\mu \nabla^{4} \psi-\eta \nabla^{6} \psi=0 .
$$

The appropriate conditions for the stream function at infinity result from the velocity far away from the sheet approaching $U$ i.e. 


$$
\left.\begin{array}{l}
\psi_{y}=U \\
\psi_{x}=0
\end{array}\right\} \text { as } y \rightarrow \infty .
$$

The differential equation (13) and boundary conditions (10-12) and (14) can be put into dimensionless form by introducing the transformations:

$$
\bar{x}=k x-\omega t, \bar{y}=k y, \bar{\psi}=\frac{k^{2}}{\omega} \psi .
$$

In view of the transformations (15) the governing problem after dropping the bars takes the form:

$$
\begin{aligned}
& \nabla^{4} \psi-\frac{1}{\alpha^{2}} \nabla^{6} \psi=0 \\
& \left.\psi_{y}\right|_{\left(x_{s}, y_{s}\right)}=\beta \varepsilon \sin x-\gamma \varepsilon \cos x, \\
& \left.\psi_{x}\right|_{\left(x_{s}, y_{s}\right)}=\varepsilon \cos x \\
& \left.\varepsilon \cos x\left(\psi_{x x x}+\psi_{x y y}\right)\right|_{\left(x_{s}, y_{s}\right)}-\left.\left(\psi_{x x y}+\psi_{y y y}\right)\right|_{\left(x_{s}, y_{s}\right)}=0 \\
& \left.\begin{array}{l}
\psi_{y}=U \\
\psi_{x}=0
\end{array}\right\} \text { as } y \rightarrow \infty,
\end{aligned}
$$

where $x_{s}=x+\varepsilon \beta \cos x+\varepsilon \gamma \sin x, y_{s}=\varepsilon \sin x, \beta=\beta^{*} / b, \gamma=\gamma^{*} / b, \varepsilon=b k$ and $\alpha=\sqrt{\mu / \eta k^{2}}$ is the couple stress fluid parameter. In the next section the governing eqn. (16) is solved subject to boundary conditions (17) and (18).

\section{PERTURBATION SOLUTION}

For the solution of the problem consisting of governing equation (16) and boundary conditions $(17,18)$ we expand the stream function and swimming velocity as:

$$
\psi=\varepsilon \psi_{1}+\varepsilon^{2} \psi_{2}+\ldots, \quad U=\varepsilon U_{1}+\varepsilon^{2} U_{2}+\ldots
$$

Substitution of expansion (19) into (16) and expanding the boundary conditions at the sheet in a Taylor series about $y=0$, we get the following system:

System of order $\varepsilon$ :

$$
\nabla^{4} \psi_{1}-\frac{1}{\alpha^{2}} \nabla^{6} \psi_{1}=0
$$




$$
\begin{aligned}
& \left.\psi_{1 y}\right|_{(x, 0)}=\beta \sin x-\gamma \cos x, \\
& \left.\psi_{1 x}\right|_{(x, 0)}=\cos x \text {, } \\
& \psi_{1 x x y}+\left.\psi_{1 y y y}\right|_{(x, 0)}=0 \text {, } \\
& \left.\begin{array}{l}
\psi_{1 y}=U_{1} \\
\psi_{1 x}=0
\end{array}\right\} \text { as } y \rightarrow \infty .
\end{aligned}
$$

System of order $\varepsilon^{2}$ :

$$
\begin{aligned}
& \nabla^{4} \psi_{2}-\frac{1}{\alpha^{2}} \nabla^{6} \psi_{2}=0 \\
& \left.\psi_{2 y}\right|_{(x, 0)}+\left.\sin x \psi_{1 y y}\right|_{(x, 0)}+\left.(\beta \cos x+\gamma \sin x) \psi_{1 x y}\right|_{(x, 0)}=0 \text {, } \\
& \left.\psi_{2 x}\right|_{(x, 0)}+\left.(\beta \cos x+\gamma \sin x) \psi_{1 x x}\right|_{(x, 0)}+\left.\sin x \psi_{1 x y}\right|_{(x, 0)}=0 \text {, } \\
& \left.\psi_{2 x x y}\right|_{(x, 0)}+\left.\psi_{2 y y y}\right|_{(x, 0)}+\left.\sin x \psi_{1 x x y y}\right|_{(x, 0)}+\left.(\beta \cos x+\gamma \sin x) \psi_{1 x x x y}\right|_{(x, 0)} \\
& +\left.\sin x \psi_{1 y y y y}\right|_{(x, 0)}+\left.(\beta \cos x+\gamma \sin x) \psi_{1 x y y y}\right|_{(x, 0)}-\left.\cos x\left(\psi_{1 x x x}+\psi_{1 x y y}\right)\right|_{(x, 0)}=0 \text {, } \\
& \left.\begin{array}{l}
\psi_{2 y}=U_{2} \\
\psi_{2 x}=0
\end{array}\right\} \text { as } y \rightarrow \infty \text {. }
\end{aligned}
$$

The solution of the leading order system yields:

$$
\begin{gathered}
\psi_{1}=\frac{1}{2-\sqrt{1+\alpha^{2}}\left(2-\alpha^{2}\right)}\left[\gamma\left\{e^{-y}\left(2-\alpha^{2} \sqrt{1+\alpha^{2}}\right)-2 e^{-\sqrt{1+\alpha^{2}} y}\right\}\right. \\
\{\cos x-(1+\beta) \sin x\}]+e^{-y} \sin x, \\
U_{1}=0
\end{gathered}
$$

Thus swimming velocity is zero at the leading order. The rate of work done by the sheet up to order $\varepsilon$ whose average value is the volume integral of $\langle\boldsymbol{\tau}: \nabla \mathbf{V}\rangle$, where $\tau$ is the total stress tensor and $\nabla \mathbf{V}$ is velocity gradient tensor, is given by the following formula:

$$
w=\frac{4 \beta\left(\alpha^{2}-1+\sqrt{1+\alpha^{2}}\right)+\alpha^{2} \sqrt{1+\alpha^{2}}\left(2+\sqrt{1+\alpha^{2}}\right)\left(1+\beta^{2}+\gamma^{2}\right)}{\left(\sqrt{1+\alpha^{2}}+\alpha^{2}-1\right)^{2}}
$$


It is evident from expression (27) that at leading order, swimming speed is independent of couple stresses $(\alpha)$. To have an estimate of swimming speed as a function of couple stress parameter $(\alpha)$ the equations at the order $\varepsilon^{2}$ are solved and it is found that:

$$
\begin{aligned}
& \psi_{2}=\left(\sqrt{4+\alpha^{2}}\left(8-\alpha^{2}\right)-16\right)^{-1}\left\{( \gamma \operatorname { s i n } 2 x + ( 1 + \beta ) \operatorname { c o s } 2 x ) \left[\frac{\alpha^{2} y e^{-2 y}}{8\left(2+\sqrt{1+\alpha^{2}}\right)}\right.\right. \\
& \left.+\left(e^{-2 y}+\frac{\alpha^{2} \sqrt{4+\alpha^{2}}}{8} y e^{-2 y}-e^{-\sqrt{4+\alpha^{2}} y}\right)\left(\frac{-\alpha^{2}}{2+\sqrt{1+\alpha^{2}}}-\frac{8 \alpha^{2}\left(\sqrt{1+\alpha^{2}}-1\right)}{2-2 \sqrt{1+\alpha^{2}}+\alpha^{2} \sqrt{1+\alpha^{2}}}\right)\right] \\
& \left.+4\left(e^{-2 y}+\frac{\alpha^{2} \sqrt{4+\alpha^{2}}}{8} y e^{-2 y}-e^{-\sqrt{4+\alpha^{2}} y}\right)\left(2 \beta \gamma \sin 2 x+\left(\beta^{2}-\gamma^{2}-1\right) \cos 2 x\right)\right\} \\
& U_{2}=\frac{\alpha^{2}\left(\sqrt{1+\alpha^{2}}-1\right)(1+\beta)}{\sqrt{1+\alpha^{2}}\left(\alpha^{2}-2\right)+2}-\frac{1}{2}\left(\beta^{2}+\gamma^{2}+1\right) .
\end{aligned}
$$

In the limiting case when $\alpha \rightarrow \infty$ we have:

$$
\begin{gathered}
U_{2}=\frac{1}{2 b^{2}}\left\{b^{2}-a^{2}+2 b \beta^{*}\right\}, \\
w=1+\frac{a^{2}}{b^{2}} .
\end{gathered}
$$

These results are in accordance with those obtained by Childress [51]. In the case when the tangential movement of the sheet is suppressed $(a=0)$ then we have:

$$
\begin{gathered}
U_{2}=\frac{\alpha^{2}\left(\sqrt{1+\alpha^{2}}-2\right)+2\left(\sqrt{1+\alpha^{2}}-1\right)}{2 \sqrt{1+\alpha^{2}}\left(\alpha^{2}-2\right)+4}, \\
w=\frac{\alpha^{2}\left(1+\alpha^{2}+2 \sqrt{1+\alpha^{2}}\right)}{\left(\sqrt{1+\alpha^{2}}+\alpha^{2}-1\right)^{2}} .
\end{gathered}
$$

When $\alpha \rightarrow \infty$, the formulae (33) and (34) in dimensional form reduce to the corresponding formulae for Newtonian swimming sheet propulsion obtained by Taylor [2], viz:

$$
U_{2}=\frac{1}{2} \omega k b^{2},
$$




$$
w=\mu \omega^{2} k b^{2} .
$$

\section{VALIDATION WITH NAKAMURA FINITE DIFFERENCE METHOD}

The boundary value problem defined by the sixth order partial differential equation (16) and boundary conditions $(17,18)$ has also been solved with an efficient second order accurate tridiagonalization algorithm developed originally by Nakamura [53]. This method is particularly adept at accommodating challenging boundary conditions as exemplified by swimming micro-organism dynamics. The Nakamura Tridiagonal Scheme (NTS) is similar to other implicit finite difference methods e.g. Keller's box method [54], but offers greater algebraic simplicity especially for two-independent variable $(x, y)$ flow problems. As with the Keller-Box scheme, higher order differential equations are reduced to a system of lower order differential equations. In recent years NTS has proved very successful in multi-physical fluid mechanics and readers may refer to recent works on non-Newtonian heat transfer [55], peristaltic propulsion [56], biopolymeric enrobing flows [57] and nanofluid bioconvection of oxytactic bacteria [58]. NTS works well for both one-dimensional (ordinary differential systems) and two-dimensional (partial differential systems) non-similar flows. NTS entails a combination of the following aspects.

(1)The flow domain for the convection field is discretized using an equi-spaced finite difference mesh in the $x$-direction.

(2)The ordinary derivatives for each respective variable (in this case stream function, $\psi$ ) with respect to $x$ are evaluated by central difference approximations.

(3)A single iteration loop based on the method of successive substitution is utilized.

(4)The finite difference discretized equations are solved as a linear second order boundary value problem of the ordinary differential equation type on the $x$-domain.

(5) An outer loop advances the solution in the $y$-direction.

The swimming equation (16) is reduced to a second order equation with an appropriate substitution. It is formulated numerically as follows:

Nakamura swimming (momentum) equation:

$$
\mathrm{A}_{1} \mathrm{~J}^{\prime \prime}+\mathrm{B}_{1} \mathrm{~J}^{\prime}+\mathrm{C}_{1} \mathrm{~J}=\mathrm{S}_{1}
$$

where $A_{i=1 \ldots 3}, B_{i=1.3}, C_{i=1.3}$ are the Nakamura matrix coefficients, $S_{i=1 \ldots 3}$ are the Nakamura source terms containing a mixture of variables and derivatives not associated with the lead variable. The 
Nakamura eqn. (37) is transformed to a finite difference equation which constitutes a tri-diagonal system that is solved iteratively. Computations take seconds on an SGI Octane desktop station. The boundary conditions $(17,18)$ are also converted to appropriate form. Double precision arithmetic is employed with Fortran 90 subroutine do-loops - see Bég [59] and Bég et al. [60]. Solutions for the swimming speed, $U$, are readily computed. We have provided comparisons with the perturbation solutions in Tables 1 and 2, for variation in couple stress parameter $(\alpha)$ and amplitude ratio $(a / b)$, respectively. Excellent correlation is achieved and therefore confidence in the present perturbation solutions (shown in all graphs) is high. To optimize the present method, the code has been tested for convergence with respect to the spatial resolution. The solutions converge in 40 iterations. Meshindependence of solutions was achieved.

\section{COMPUTATIONS AND DISCUSSION}

This section displays some graphical results in order to see the effects of couple stress fluid parameter $(\alpha)$ on swimming speed $\left(U_{2}\right)$ and rate of work done by the sheet $(w)$. It has been pointed by Stokes [34] that the effects of couple stresses are quite large for small values of $\alpha=\sqrt{\mu / \eta} / k$, where $1 / k$ is a typical length scale associated with the flow and $\sqrt{\mu / \eta}$ is a material constant which is a function of molecular dimensions of the liquid. For large values of $(\alpha)$ the effects of couple stresses diminish and fluid tends to a Newtonian liquid. Since the parameters $a / b$ and $\phi$ control the beat pattern of the sheet therefore the beat patterns of the sheet for different values of $\phi$ and $a / b$ are shown in Figs. 2ad.

It is observed that the stretching wave at the sheet surface slightly modifies the normal beat pattern of the sheet for small values of $a / b$. This normal beat pattern corresponds to the beat pattern of the sheet when there is no stretching wave. A considerable modification in the normal beating pattern occurs when the amplitude of the stretching wave begins to increase (Fig. 3a). Large values of $a / b$ produce beat patterns which may be physically unrealistic owing to the appearance of a cusp. Fig. 2(a) shows the beat pattern of the sheet for different values of $a / b$ when $\phi=0$ and the corresponding swimming speed plotted against $\alpha$ for these beat patterns can be seen through Fig. 4(a). We can observe from Fig. 3(a) that the beat pattern corresponding to $a / b=0.5$ and 1 produces larger swimming speeds than the normal beat pattern for all values of $\alpha$. It is further observed that the beat pattern corresponding to $a / b=1.5$ is not effective for small values of $\alpha$. However, it becomes effective compared to the normal beat pattern for values of $\alpha$ greater than 1.7. The beat patterns of the sheet for different values 
of $a / b$ when $\phi=\pi / 6$ are displayed in Fig. 2(b). The swimming speeds corresponding to these beat patterns are shown in Fig. 4(b). Again we note that beat patterns for $a / b=0.5$ and 1 are more beneficial than the normal beat pattern and beat pattern for $a / b=1.5$ and become effective when $\alpha>5$. The beat pattern corresponding to $\phi=\pi / 4$ and $\pi / 3$ are not shown because they do not differ much from the beat patterns for $\phi=0$ and $\pi / 6$. However, the swimming speeds corresponding to these beat patterns are depicted in Figs. 4(c) and 4(d). From both figures we note that beat pattern corresponding to $a / b=0.5$ is more efficient than the normal beat pattern.

Fig. 3(c) shows that when $\phi=\pi / 4$ the beat pattern for $a / b=1$ is better than normal beat pattern after $\alpha=0.9$. As $\phi$ increase from $\pi / 4$ to $\pi / 2$ the only effective beat pattern is for $a / b=0.5$. A further increase in $\phi$ reveals that the normal beat pattern performs best compared with other beat patterns. These observations indicate that normal beat pattern is effective for both couple stress and Newtonian fluids only when the amplitude of the stretching wave is small. Furthermore, plausible values of $a / b$ and $\phi$ may be selected to achieve a beat stroke which can attain greater swimming speed both for couple stress and Newtonian fluids compared to the normal beat stroke scenario. It is also interesting to note from Figs. 4a-f that the swimming speed of the sheet decreases with couple stress effect $(\alpha)$ and reaches asymptotically to a constant value as $\alpha \rightarrow \infty$. Thus the presence of suspended particles in a viscous fluid reduces the swimming speed of micro-organisms. In other words swimming speed of a micro-organism in Newtonian liquid is higher than in couple stress fluid. This behavior agrees with other non-Newtonian (viscoelastic) models reported in literature $[\mathbf{2 8}, \mathbf{3 1}]$ where it has also been shown that visoelasticity tends to decrease swimming speed. However it deviates from the micropolar model [e.g. 27] results wherein, contrary to couple stress models, with greater micropolar viscosity effect (stronger rheological behaviour), propulsive velocity is increased. It is suggested that this acceleration is attributable in the micropolar model to the intrinsic spin of micro-elements via gyro-rotary motions [61] and this mechanism is absent from the Stokesian couple stress model, since the latter does bit possess micro-structure at the kinematic level and this is the primary cause for achieving flow acceleration (and drag reduction) in micropolar liquids [60]. The couple stress model, exhibiting limited micro-structure, effectively correlates more closely with viscoelastic (Oldroyd-B models) than micro-morphic rheological models. The lower complexity of the couple stress model relative to micropolar models is indicated by the supplementary sixth order derivatives appearing in the modified stream function eqn. (16)- this term when neglected reduces eqn. (16) to the Taylor (Newtonian) 
model. In micropolar models, separate balance equations arise which result in an interaction between the linear velocity field (sheet) and the angular velocity field (micro-element gyration). This level of sophistication simply cannot be captured by couple stress models. Nevertheless the present couple stress model does provide an improvement to the conventional viscoelastic formulations and is essentially a bridge between viscoelastic non-Newtonian models and the more refined microcontinuum models of Eringen. Cowin [52] has elaborated in some detail, that larger values of couple stress parameter, $(\alpha)$, basically elevates the global viscosity of the couple stress fluid which induces flow deceleration. The micropolar model however has a different viscosity mechanism (vortex viscosity- associated with spin), which provides a more complex framework than couple stress theory. A secondary objective of the present work is to open up the discussion and interest in generalized micro-structural swimming hydrodynamics and stimulate more interest in the use of these models, which could identify other mechanisms and features not achievable with the viscoelastic family of models.

Fig. 5 delineates the effects of amplitude ratio $a / b$ and phase difference $\phi$ on swimming speed for fixed values of couple stress parameter $(\alpha)$. From this figure one can find the (critical) value of $a / b$ below which $U_{2}$ is positive and above which it is negative. We recall that positive $U_{2}$ means that the sheet swims to left and vice versa. It can been seen from Fig. 3 that for $\phi=0$ and $\alpha=2$, swimming speed (propulsive velocity) becomes negative when $a / b$ exceeds 1.8 . However, $a / b$ must be less than 0.7 for $U_{2}$ to be positive when $\phi=\pi / 2$ and $\alpha=2$. Similarly for $\phi=\pi$ and $\alpha=2, a / b$ should be less than 0.3 for $U_{2}$ to be positive. The above observation implies that the critical value of $a / b$ decreases with $\phi$. A similar observation for Oldroyd-B fluid swimmers has been reported in Teran et al. [32]. The study [32] also confirms the correlation in results between the present couple stress model and simpler viscoelastic models, the latter also exhibiting deceleration in swimming speeds with nonNewtonian (viscoelastic effects) relative to a Stokesian Newtonian fluid. However in [32] it is also identifed that for finite micro-organism sheets - the so-called swimming "free" sheets (i.e., with free head and tail), greater propulsive swimming velocity is achieved with stronger viscoelastic effects (Deborah numbers of order unity) relative to Newtonian fluids, proposing that this may be due to the relaxation time equivalence with the stroke frequency of the swimmer (as a result of zones of highly strained viscoelastic fluid located aft of the free swimmer's tail which suppress reverse slippage and thereby increasing the average speed). The couple stress model may therefore be more representative for real swimmers since it does indeed predict acceleration in the swimming speed. Propulsive 
performance in [32] and indeed other non-Newtonian simulations $[\mathbf{2 7}, \mathbf{3 7}]$ is found to be intimately associated with amplitude ratio and phase difference, again adding confidence to the present computations. The degree of sensitivity however notably varies with the nature of the non-Newtonian model. It would appear therefore of interest to extend the present couple stress infinite sheet model to a finite free head-tail geometric model in the future to evaluate more precisely whether couple stresses exert effects more consistent with clinical observations than viscoelastic models, for this configuration, and this is under consideration by the authors. It is further noted from Fig. 5 that critical value of $a / b$ increase with $\alpha$.

Fig. 6 depicts the rate of work done by the sheet as a function of $\alpha$, plotted for different values of $a / b$. This figure reveals that rate of work done decreases by increasing $\alpha$. Physically this is consistent with the decrease in swimming sheet speed with greater couple stress parameter since greater work is expended to achieve propulsion of the micro-organism as non-Newtonian effects are enhanced. Propulsion in couple-stress fluids is therefore demonstrably less efficient than in Newtonian fluids or viscoelastic fluids. Again this observation deviates from the micropolar sheet model computations of Sinha et al. [26] and Philip and Chandra [27], demonstrating that both models have some promise in spermatozoa hydrodynamics. Fig. 6 also indicates an increase in the rate of work done by the sheet with an increase in $a / b$ (amplitude ratio) and this is however, in agreement with both micropolar [26, 27] and other viscoelastic studies of swimming sheets $[\mathbf{2 8 , 3 1}$.

\section{CONCLUSIONS}

The problem of an infinite flexible sheet swimming in a couple stress rheological biofluid is analyzed, as a model of spermatozoa dynamics in embryological transport. The mathematical model presented is a significant and novel extension of classical Taylor's swimming sheet problem (for Newtonian viscous fluids). The conservation equations are transformed via a stream function formulation, and the resulting sixth order homogenous partial differential equation is solved with a perturbation method (series solution) up to second order in terms of the dimensionless amplitude of the normal motion of the sheet (perturbation parameter). The emerging formulae for swimming speed and rate of work done by the sheet are obtained and are strong functions of the couple stress fluid parameter. Validation of the perturbation solutions is achieved with a Nakamura tridiagonal finite difference simulation (NTS). Some interesting features regarding swimming speed and rate of work done by the sheet are highlighted through graphical results. The main observation of the present analysis is that swimming effort made by micro-organism increases whereas swimming (propulsive) speed decreases as the fluid 
changes from Newtonian to couple stress fluid. This observation may have some biological implications and indeed better concurs with some clinical observations. As pointed out by Lauga [28], the use of non-Newtonian fluids to study locomotion of micro-organisms is more appropriate because their rheological properties can be utilized to manipulate the swimming speed instead of altering the motion of micro-organism which is the only possibility if the fluid considered is Newtonian. The sophistication afforded by micro-structural models (Stokes couple stress [38], Eringen's micropolar model [39]) however is significantly greater than those considered by conventional viscoelastic models which neglect body couples and important micro-rheological characteristics associated with actual embryological fluids. Since the cervical mucus is a suspension of long chain molecules and presence of these molecules produce couple stresses, this mechanism may be a primary cause affecting propulsive efficiency. The presence of micro-structure (although not fully simulated with couple stress fluids) in the present model does relate better however to mucus fluid in embryological systems being plausible for locomotion. The second main finding of the present study is that the presence of a stretching wave on the sheet produces beat strokes which are more efficient than the beat stroke in the absence of this wave. This observation may be helpful in the design of artificial micro-robotic swimmers for medical treatment. Overall the present theoretical investigation has identified important fluid dynamic characteristics of couple stresses which encourages the exploration of micro-continuum hydrodynamics as a more robust approach in understanding the motion of micro-organism in complex fluids (e.g. reproductive) and in this regard it is hoped the present work will stimulate greater interest among researchers to employ such models. The present work has neglected global rotational effects (wherein the entire medium is rotating) and entropy generation aspects. In this regard important benchmarks for future simulation would be the works of [62]-[65] and efforts in these directions are currently being considered.

\section{ACKNOWLEDGEMENTS}

The authors are extremely grateful to both reviewers for their excellent comments which have served to improve the article.

\section{REFERENCES}

[1]M. Murase, The Dynamics of Cellular Motility, John Wiley, Chichester, UK (1992).

[2]G. I. Taylor, Analysis of the swimming of microscopic organisms, Proc. R. Soc. Lond. A 209, 447461 (1951). 
[3]G. I. Taylor, The action of waving cylindrical tails in propelling microscopic organisms, Proc. $R$. Soc. Lond. A, 211, 225-239 (1952).

[4] G. J. Hancock, The self-propulsion of microscopic organisms through liquids, Proc. R. Soc. Lond. A, 217, 96-121 (1953).

[5] J. Gray and G. J. Hancock, The propulsion of sea-urchin spermatozoa, J. Exp. Biol. 32, 802-814 (1955).

[6] M. E. J. Holwill, Physical aspects of flagellar movement, Physiol. Rev., 46, 696-785 (1966).

[7] M. E. J. Holwill and M. A. Sleigh, Propulsion by hispid flagella, J. Exp. Biol., 47, 267-276 (1967).

[8] C. J. Brokaw, Computer simulation of flagellar movement. I. Demonstration of stable bend propagation and bend initiation by the sliding filament model. Biophys. J. 12, 564-586 (1972).

[9] J. E. Drummond, Propulsion by oscillating sheets and tubes in a viscous fluid, J. Fluid Mech., 25, 787-793 (1966).

[10] A. J. Reynolds, The swimming of minute organisms, J. Fluid Mech. 23, 241-260 (1965).

[11] E. O. Tuck, A note on swimming problem, J. Fluid Mech. 31, 305-308 (1968).

[12] S. Childress, Intertial swimming as a singular perturbation, Proc. ASME 2008 Dynamic System and Control Conference, Ann Arbor, Michigan, USA, 20-22 October (2008).

[13] J. R. Blake, Infinite models for ciliary propulsion, J. Fluid Mech. 49, 209-222 (1971).

[14] C. Brennen, An oscillating boundary-layer theory of ciliary propulsion, J. Fluid Mech., 65, 799 824 (1974).

[15] C. Brennen and H. Winet, Fluid mechanics of propulsion by cilia and flagella, Ann. Rev. Fluid Mech., 9, 339-398 (1977).

[16] B. U. Felderhof, Swimming and peristaltic pumping between two plane parallel walls, J. Phys.: Condens. Matter, 21204106 (2009).

[17] M. Jaffrin and A. Shapiro, Peristaltic pumping, Ann. Rev. Fluid Mech. 3, 13-36 (1971).

[18] L. J. Fauci and R. Dillon, Biofluid mechanics of reproduction. Ann. Rev. Fluid Mech. 38, 371-394 (2006).

[19] O. S. Pak and E. Lauga, The transient swimming of a waving sheet, Proc. R. Soc. Lond. A, 466, 107-126 (2010).

[20] W. J. Shack and T. J. Lardner, A long wavelength solution for a microorganism swimming in a channel, Bull. Math. Biol., 36, 435-444 (1974). 
[21] R. E. Smelser, W. J. Shack and T. J. Lardner, The swimming of spermatozoa in an active channel. J. Biomech. 7, 349-355 (1974).

[22] D. F. Katz, On the propulsion of micro-organisms near solid boundaries, J. Fluid Mech. 64, 33-49 (1974).

[23] J. B. Shukla, B. R. P. Rao and R. S. Parihar, Swimming of spermatozoa in cervix: effects of dynamical interaction and peripheral layer viscosity, J. Biomech. 11, 15-19 (1978).

[24]J. B. Shukla, P. Chandra and R. Sharma, Effects of peristaltic and longitudinal wave motion of the channel wall of movement of micro-organisms: application to spermatozoa transport, J. Biomech. 21, 947-954 (1988).

[25] T. K. Chaudhury, On swimming in a viscoelastic liquid, J. Fluid Mech., 95, 189-197 (1979).

[26] P. Sinha, C. Singh and K. R. Prasad, A micro-continuum analysis of the self-propulsion of the spermatozoa in the cervical canal, Int. J. Eng. Sci., 20, 1037-1048 (1982).

[27] D. Philip and P. Chandra, Self-propulsion of spermatozoa in microcontinua: effects of transverse wave motion of channel walls, Arch. Appl. Mech., 66, 90-99 (1995).

[28] E. Lauga, Propulsion in a viscoelastic fluid, Phys. Fluids, 19, 083104 (2007).

[29] G. J. Elfring, O. S. Pak and E. Lauga, Two-dimensional flagellar synchronization in viscoelastic fluids, J. Fluid Mech., 646, 505-515 (2010).

[30] H. C. Fu, C. W. Wolgemuth and T. R. Powers, Swimming speeds of filaments in nonlinearly viscoelastic fluids, Phys. Fluids, 21, 033102 (2009).

[31] H. C. Fu and T. R. Powers, Theory of swimming filaments in viscoelastic media, Phys. Rev. Lett., 99, 258101 (2007).

[32] J. Teran, L. Facui and M. Shelley, Viscoelastic response can increase the speed and efficiency of a free swimmer, Phys. Rev. Lett., 104, 038101 (2010).

[33] N. J. Balmforth, D. Coombs and S. Pachmann, Microelastohydrodynamics of swimming organisms near solid boundaries in complex fluids, Q. J. Mech. Appl. Math., 63, 267-294 (2010).

[34] E. E. Riley and E. Lauga, Enhanced active swimming in viscoplastic fluids, Euro. Phys. Lett. 108, 34003 (2014).

[35] R. Arco, J. R. Velez-Cordero, E. Lauga, and R. Zenit, Viscous pumping inspired by flexible propulsion, Bioinspir. Biomim., 9, 036007 (2014).

[36] L. Zhu, E. Lauga and L. Brandt, Self-propulsion in viscoelastic fluids: pushers vs. pullers, Phys. Fluids, 24, 051902, 2012. 
[37] E. E. Riley and E. Lauga, Small-amplitude swimmers can self-propel faster in viscoelastic fluids, J. Theor. Biol., 382, 345-355 ( 2015).

[38] V. K. Stokes, Couple stress in fluids, Phys. Fluids, 9, 1709-1715 (1966).

[39] A.C. Eringen, Micro-Continuum Field Theories II-Fluent Media, Springer, New York (2001).

[40] D. Srinivasacharya and K. Kaladher, Mixed convection in a couple stress fluid with Soret and Dufour effects, Int. J. Appl. Math. Mech., 7, 59-71 (2011).

[41] L. M. Srivastava, Peristaltic transport of a couple-stress fluid, Rheol. Acta, 25, 638-641 (1986).

[42] E. F. El-Shehawey, K.S. Mekheimer, Couple-stresses in peristaltic transport of fluids, J. Phys. D. Appl. Phys. 27, 1163-1170 (1994).

[43] N. Ali, T. Hayat and M. Sajid, Peristaltic flow of a couple stress fluid in an asymmetric channel, Biorheology, 44, 125-138 (2007).

[44] D. Tripathi and O. Anwar Bég, Transient magneto-peristaltic flow of couple stress biofluids: a magneto-hydro-dynamical study on digestive transport phenomena, Mathematical Biosciences, 246(1):72-83 (2013).

[45] D. Pal, N. Rudraiah, R. Devanathan, A couple stress model of blood flow in the microcirculation, Bull. Math. Biol. 50, 329-344 (1988).

[46] O. Anwar Bég, S.K. Ghosh, S. Ahmed and T. A. Bég, Mathematical modelling of oscillatory magneto-convection of a couple stress biofluid in an inclined rotating channel, J. Mechanics In Medicine and Biology, 12, 1250050.1-1250050.35 (2012).

[47] J. Zueco and O. Anwar Bég, Network numerical simulation applied to pulsatile non-Newtonian flow through a channel with couple stress and wall mass flux effects, Int. J. Applied Mathematics and Mechanics, 5, 1-16 (2009).

[48] M. Nabhani, M. El Khilfi and B. Bou-Said, Non-Newtonian couple stress poroelastic squeeze film, Tribology Int., 54, 116-127 (2013).

[49] E. Odeblad, Undulations of macromolecules in cervical mucus, Int. J. Fertil. 7, 313-9 (1962).

[50] M. Elstein, R. F. Mitchell and J. T. Syrett, Ultrastructure of cervical mucus, J. Obstet. Gynaec. Br. Commonw. 78, 180-183 (1971).

[51] S. Childress, Mechanics of Swimming and Flying, Cambridge University Press, USA, pp 22-27 (1981).

[52] S.C. Cowin, The theory of polar fluids, Adv. Appl. Mech., 14, 279-347 (1974). 
[53] Nakamura, S, Iterative finite difference schemes for similar and nonsimilar boundary layer equations, Advances in Engineering Software, 21: 123-130 (1994).

[54] S. Nakamura, Applied Numerical Methods and Software, Prentice-Hall, New Jersey (1995).

[55] O. Anwar Bég, Bég TA, Takhar HS and Raptis A, Mathematical and numerical modeling of nonNewtonian thermo-hydrodynamic flow in non-Darcy porous media, Int. J. Fluid Mechanics Research, 31: 1-12 (2004).

[56] D. Tripathi and O. Anwar Bég, Mathematica numerical simulation of peristaltic biophysical transport of a fractional viscoelastic fluid through an inclined cylindrical tube, Computer Methods in Biomechanics and Biomedical Engineering, 18, 1648-1657 (2015).

[57] O. Anwar Bég, J. Zueco, M. Norouzi, M. Davoodi, A. A. Joneidi, Assma F. Elsayed, Network and Nakamura tridiagonal computational simulation of electrically-conducting biopolymer micro-morphic transport phenomena, Computers in Biology and Medicine, 44, 44-56 (2014).

[58] O. Anwar Bég, V.R. Prasad, B. Vasu, Numerical study of mixed bioconvection in porous media saturated with nanofluid and containing oxytactic micro-organisms, J. Mechanics Medicine Biology, 13, 1350067.1-1350067.25 (2013).

[59] O. Anwar Bég, Nakamura algorithms for biofluid dynamics, Technical Report BIO-341/March, 114 pages, Gort Engovation, Bradford, UK and Narvik, Norway, March (2013).

[60] O. Anwar Bég, R. Bhargava and M.M. Rashidi, Numerical Simulation in Micropolar Fluid Dynamics, Lambert Academic Publishing, Saarbrucken, Germany, 288 pages (2011).

[61] G. Ravi Kiran, G. Radhakrishnamacharya and O. Anwar Bég, Peristaltic flow and hydrodynamic dispersion of a reactive micropolar fluid: simulation of chemical effects in the digestive process, $J$. Mechanics in Medicine and Biology (2016). DOI: 10.1142/S0219519417500130 (17 Pages)

[62] P. Aparna, J.V. Ramana Murthy and G. Nagaraju, Couple on a rotating permeable sphere in a couple stress fluid, Ain Shams Engineering Journal (2016). doi:10.1016/j.asej.2016.03.012

[63] R. M. Josyula,S. Jangili, K.S. Sai, Flow of two immiscible couple stress fluids between two permeable beds, J.Porous Media 17(4):287-300 (2014).

[64] G. Nagaraju, S. Jangili, R. M. Josyula, A. M. Rashad, Entropy generation analysis of the MHD flow of couple stress fluid between two concentric rotating cylinders with Porous Lining, Heat Transfer-Asian Research (2016). DOI: 10.1002/htj.21214

[65] R.M. Josyula and S. Jangili, First and second law analysis for the MHD flow of two immiscible couple stress fluids between two parallel plates, Heat Transfer-Asian Research 44(5):468-487 (2014). 


\section{FIGURES}

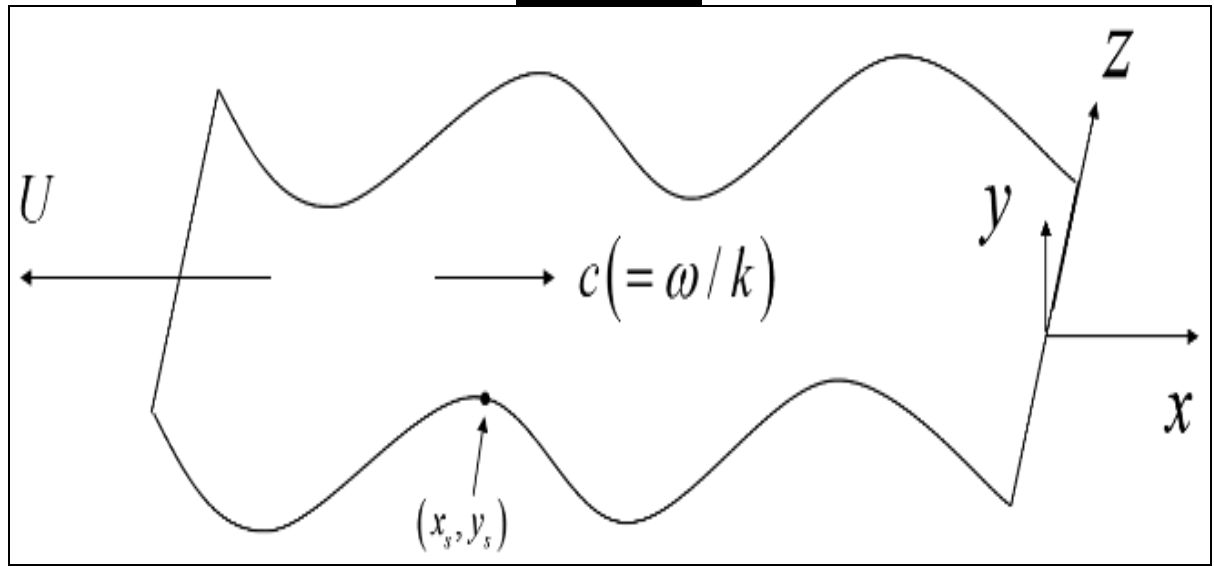

Fig.1: Schematic diagram of a swimming sheet in couple stress fluid.

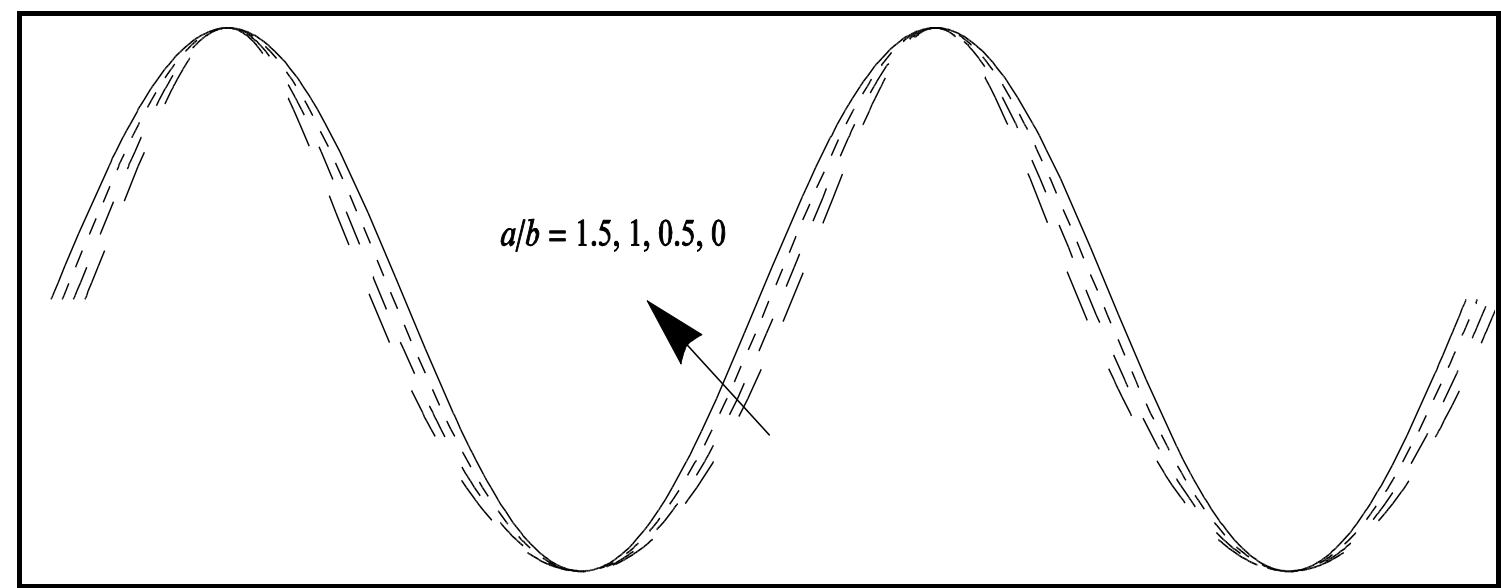

(a)

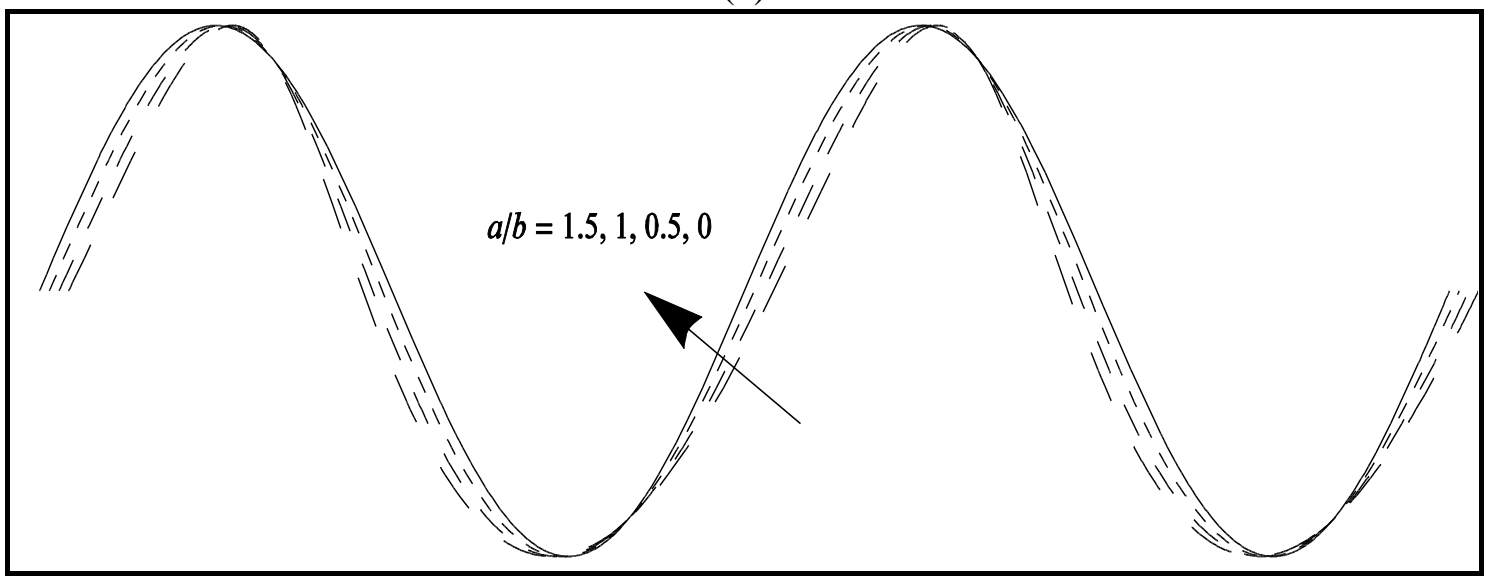

(b) 


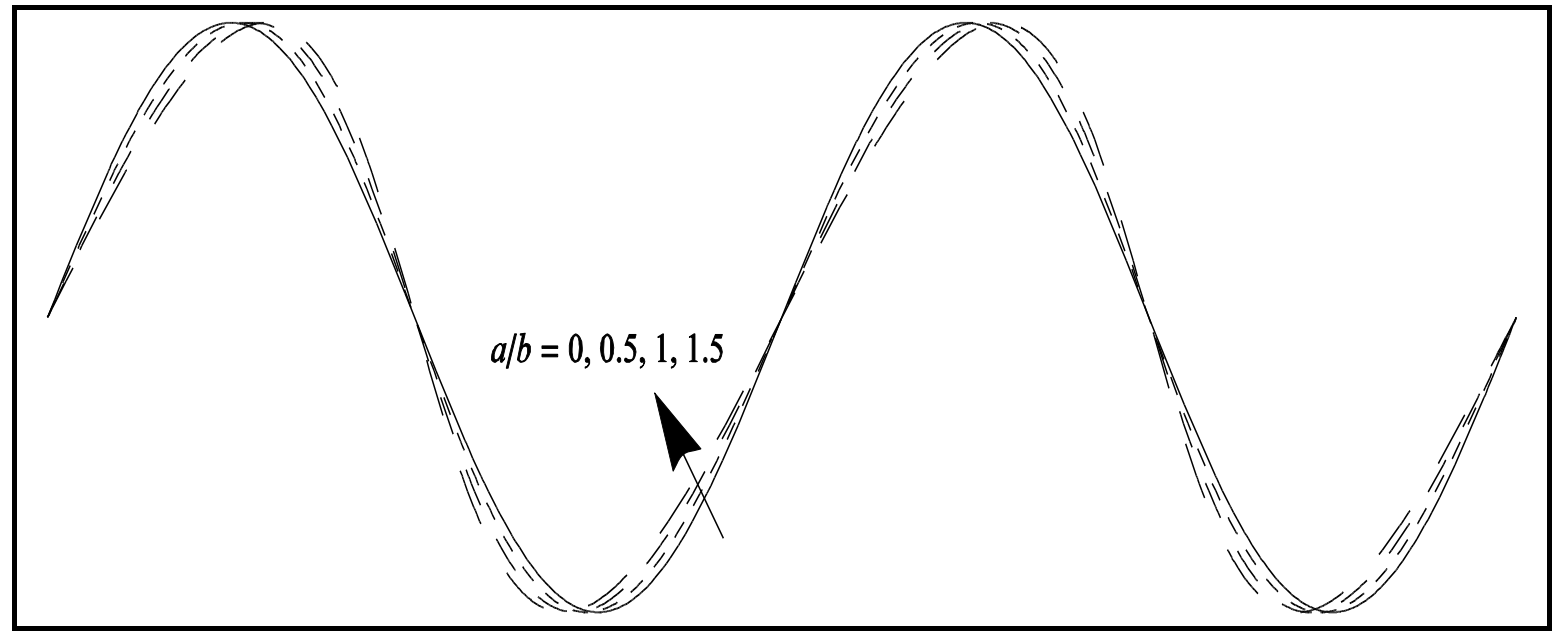

(c)

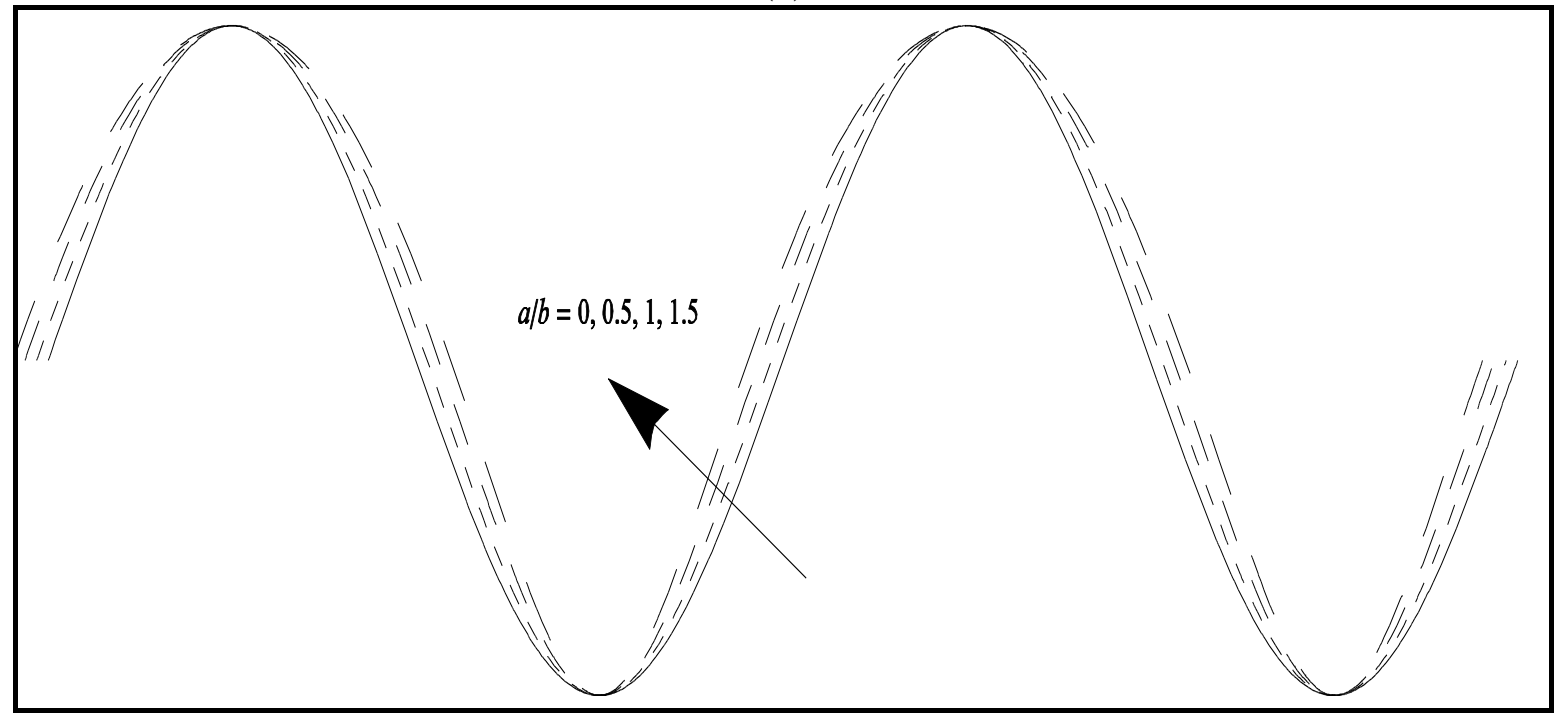

(d)

Fig. 2: Beat patterns of the sheet for different values of $a / b$. Panels (a-d) correspond to $\phi=0, \pi / 6, \pi / 2, \pi$, respectively.

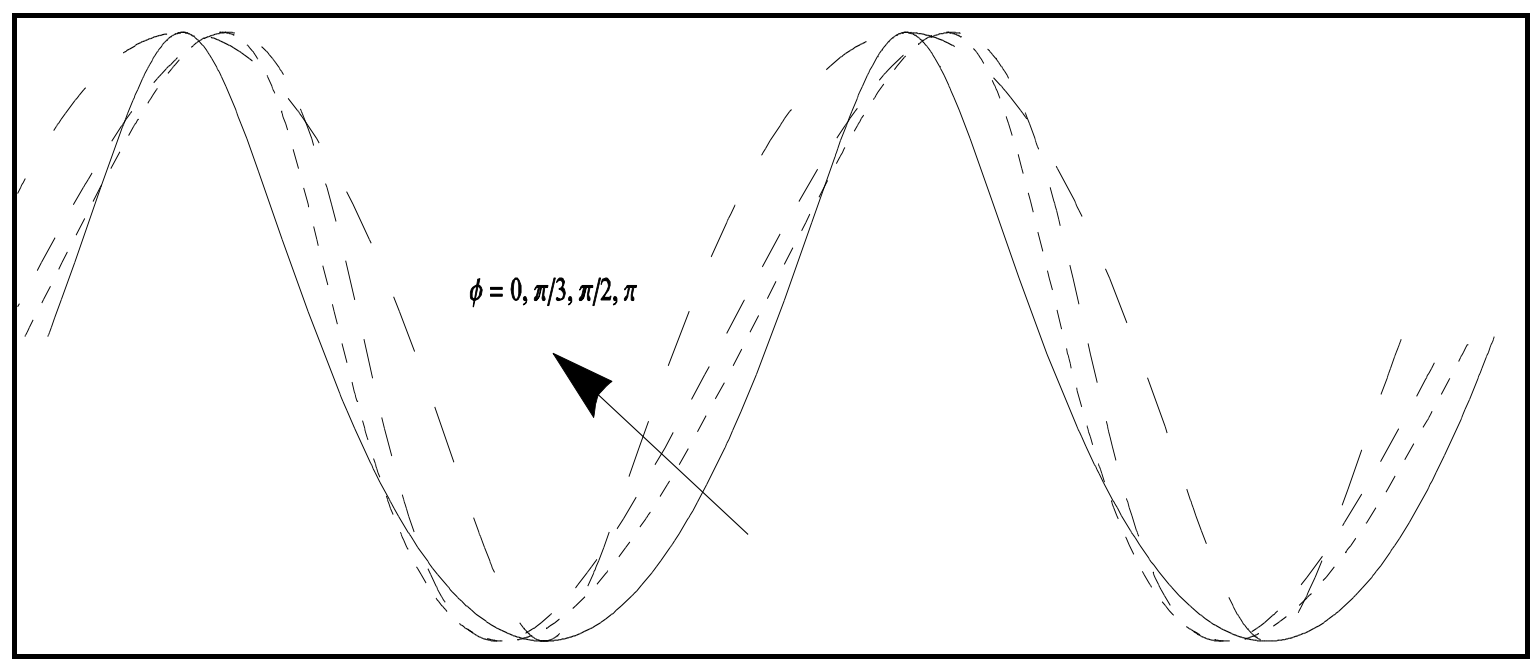

(a) 


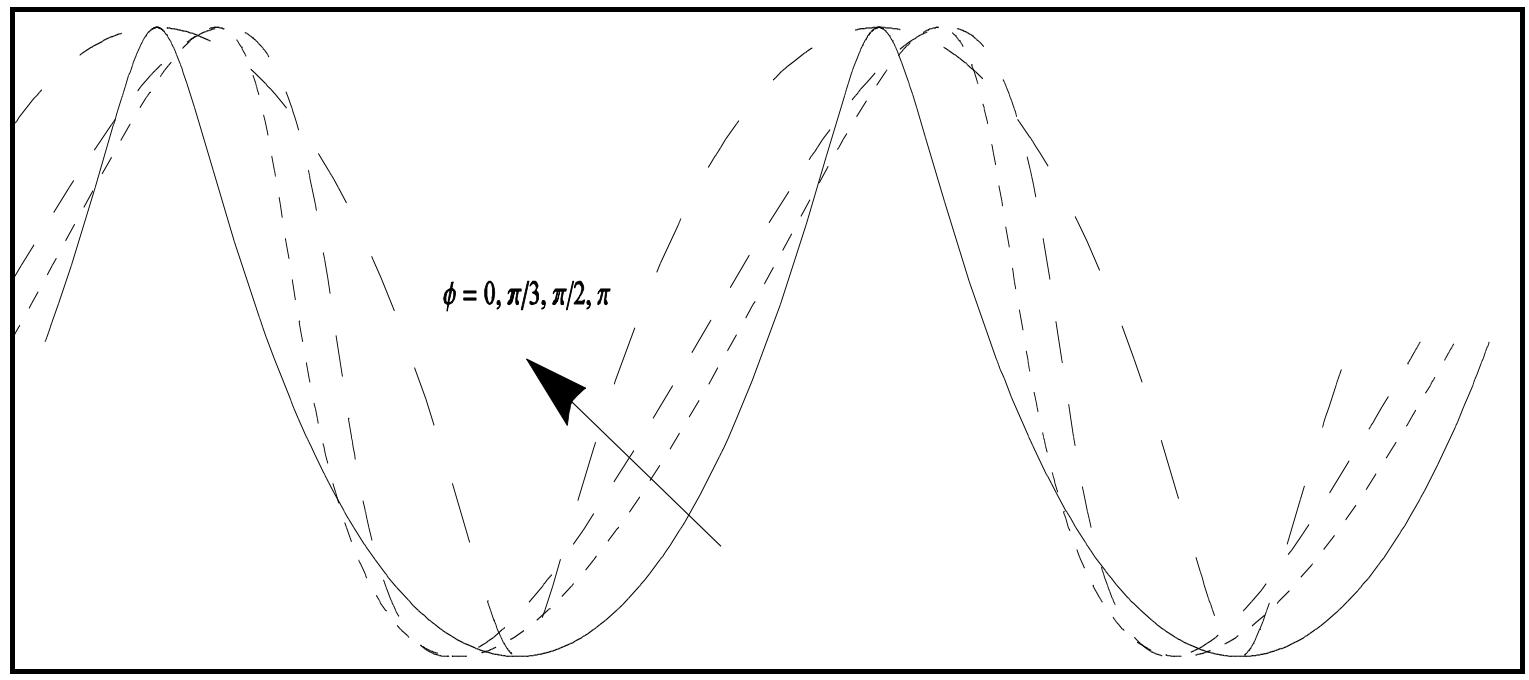

(b)

Fig. 3: Beat patterns of the sheet for different values of $\phi$. In panel (a) $a / b=2$ while in panel (b) $a / b=3$.

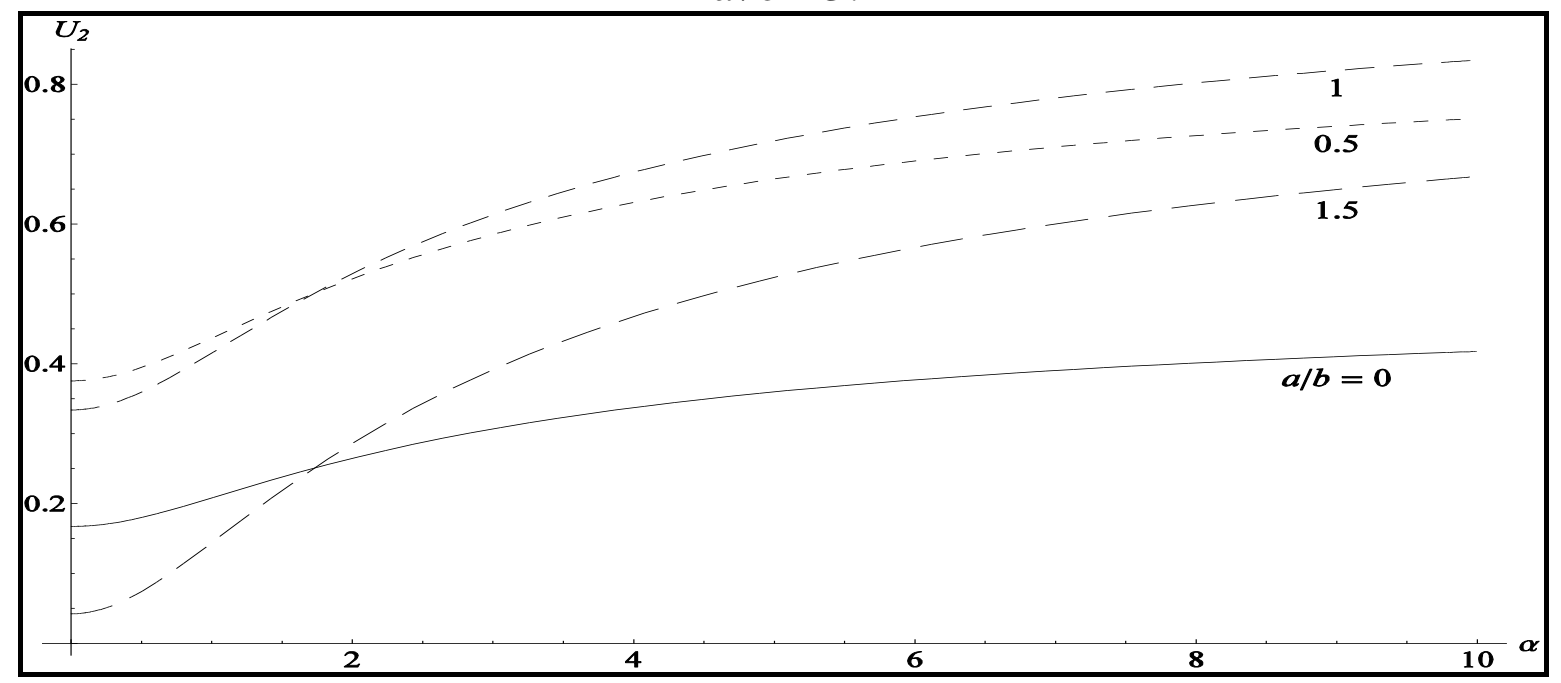

(a)

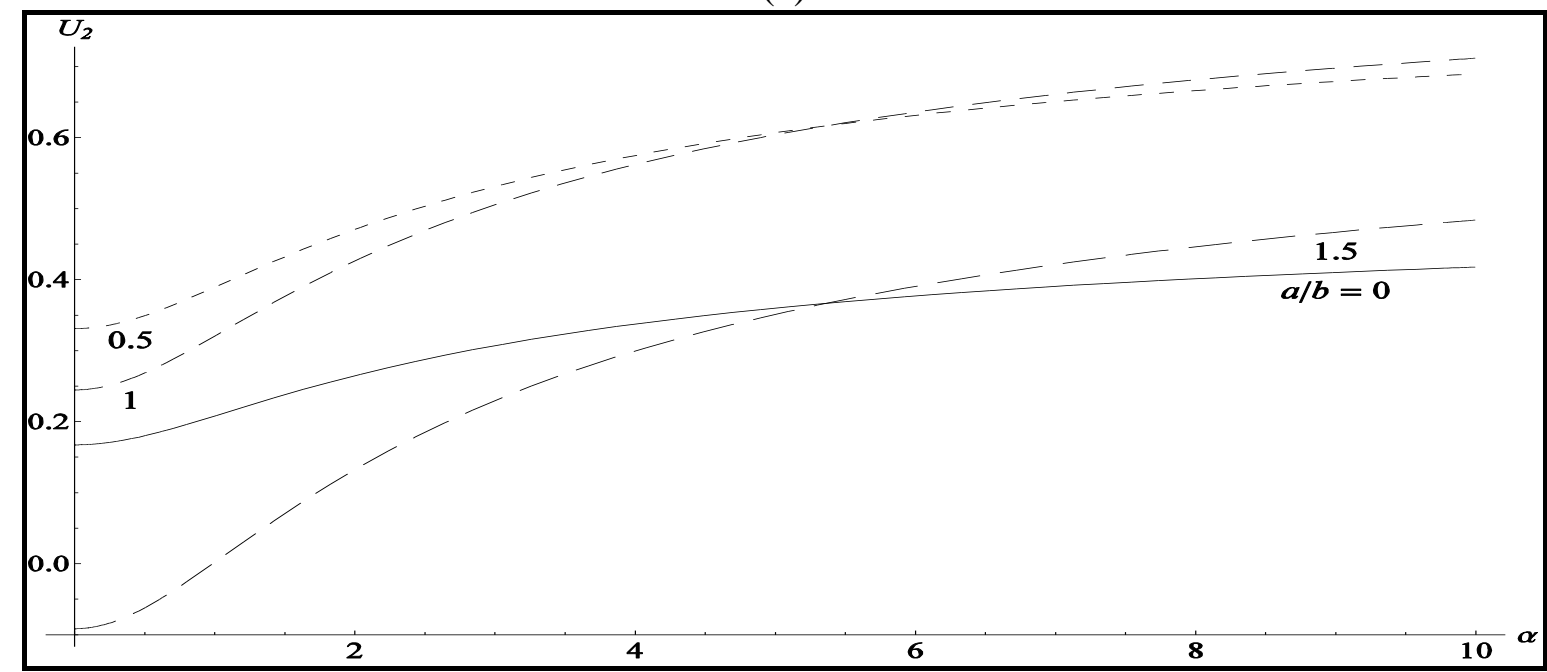

(b) 


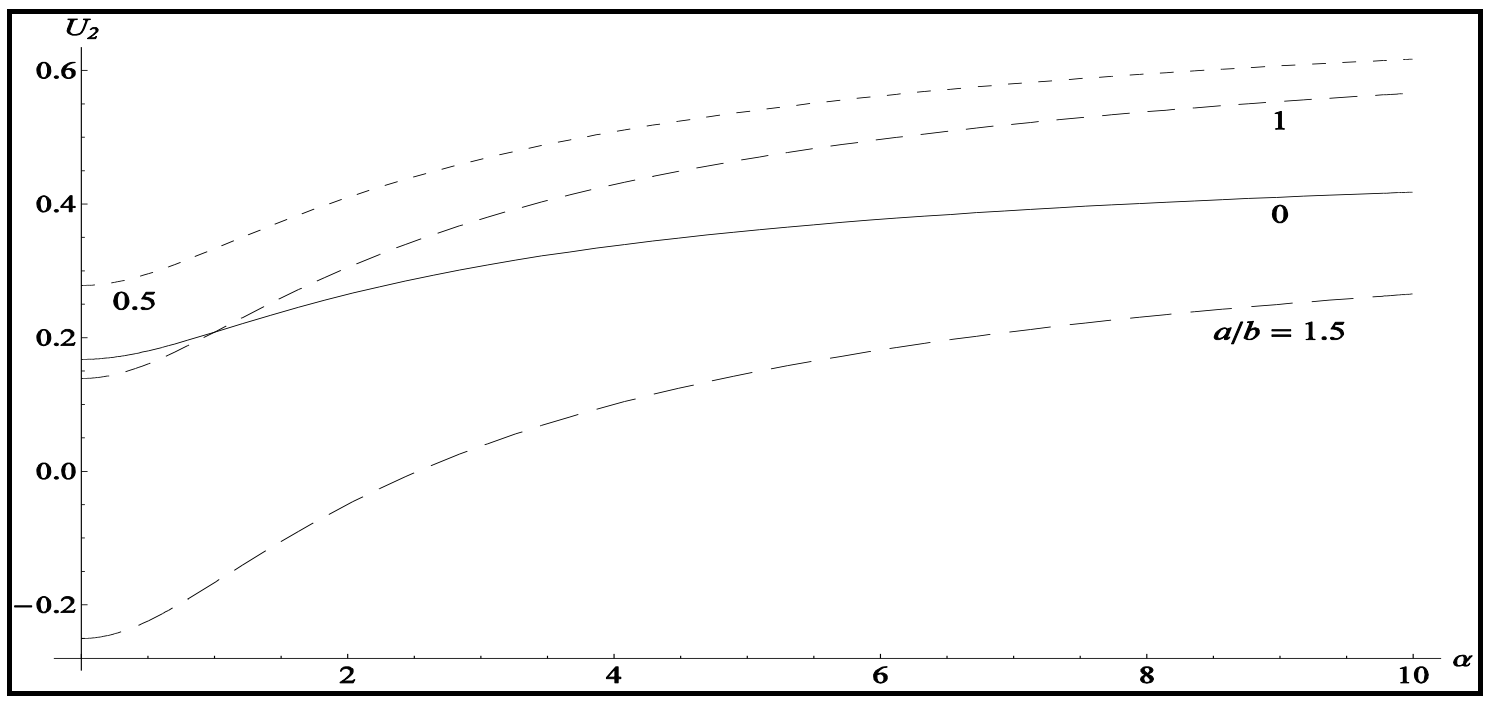

(c)

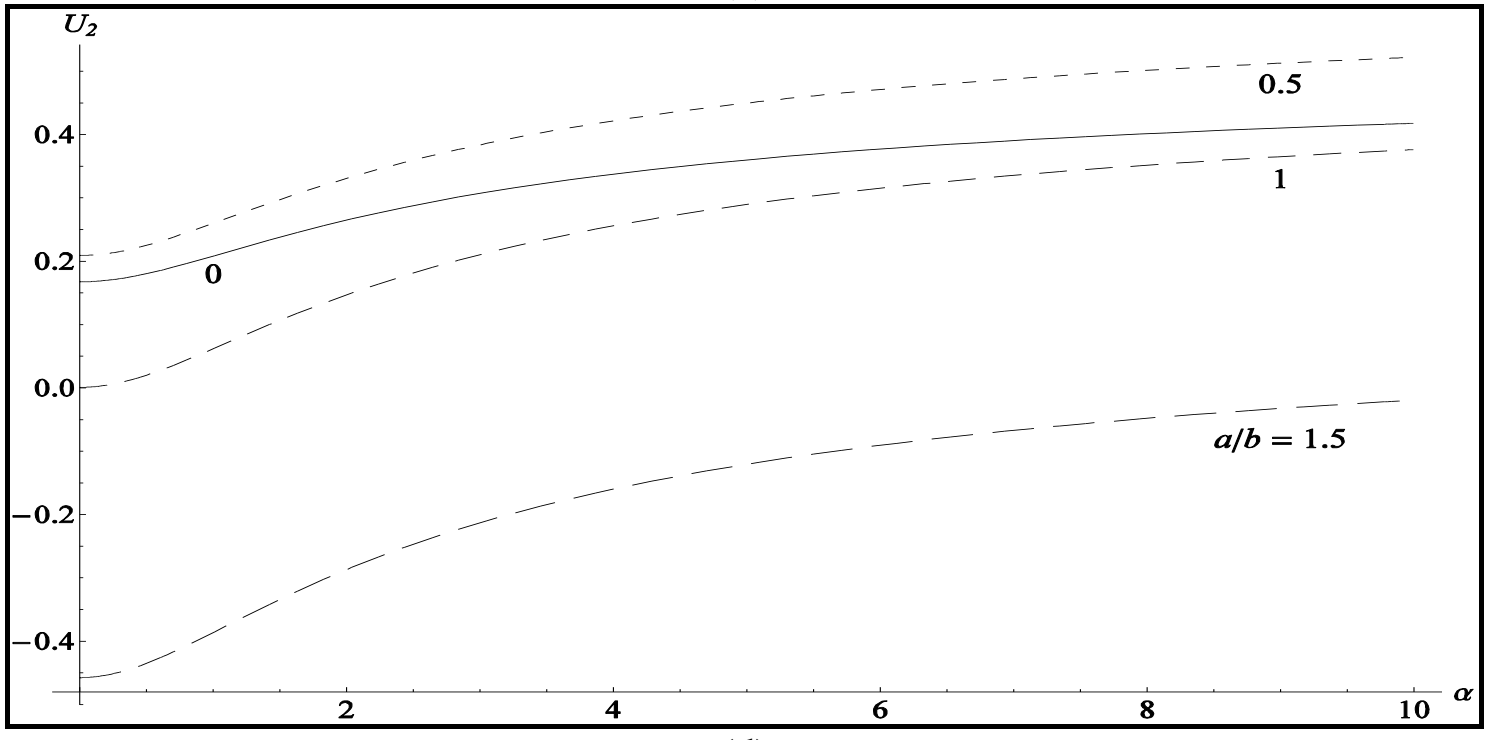

(d)

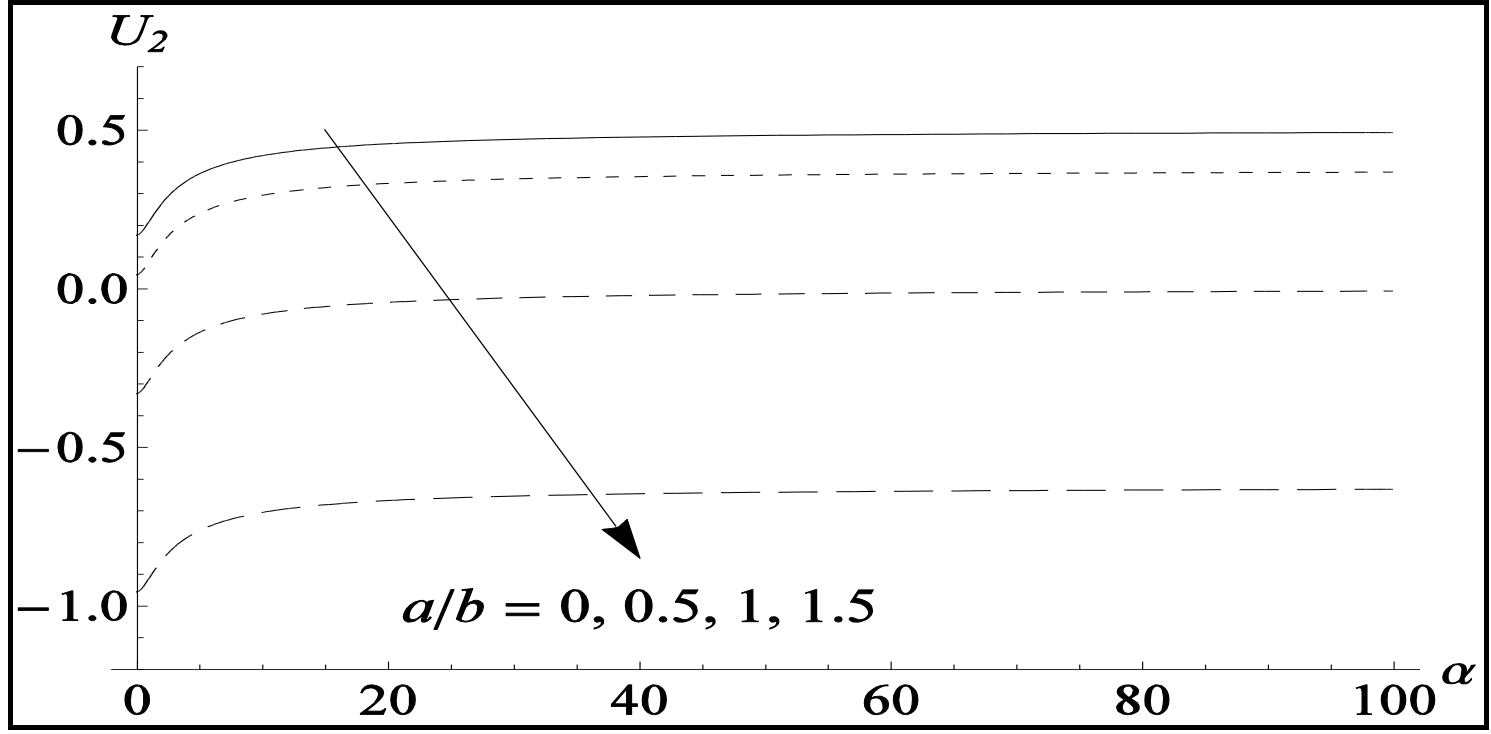

(e) 


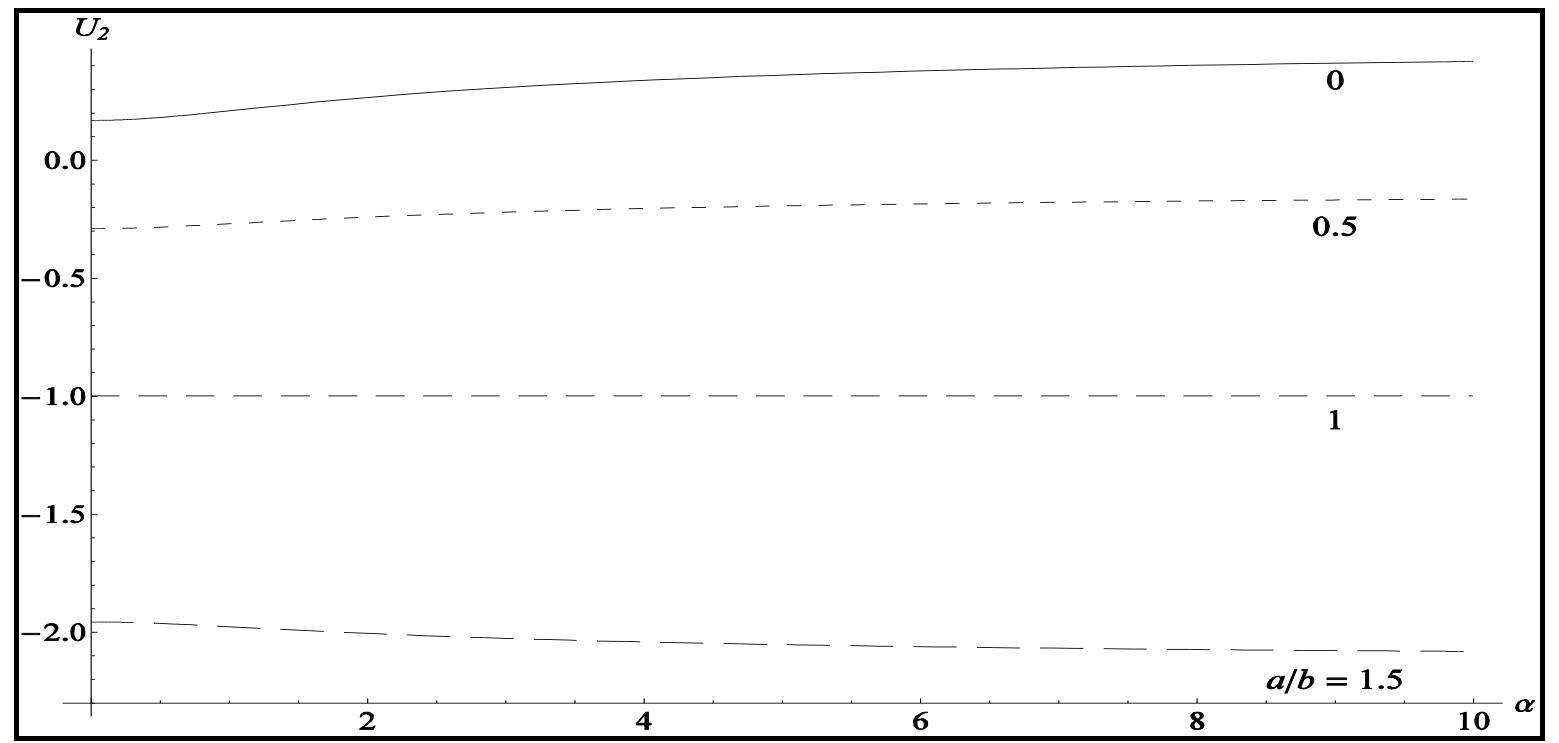

(f)

Fig. 4: Swimming speed as a function of couple stress fluid parameter $\alpha$ for different values of $a / b$. Panels (a-f) correspond to $\phi=0, \pi / 6, \pi / 4, \pi / 3, \pi / 2, \pi$, respectively.

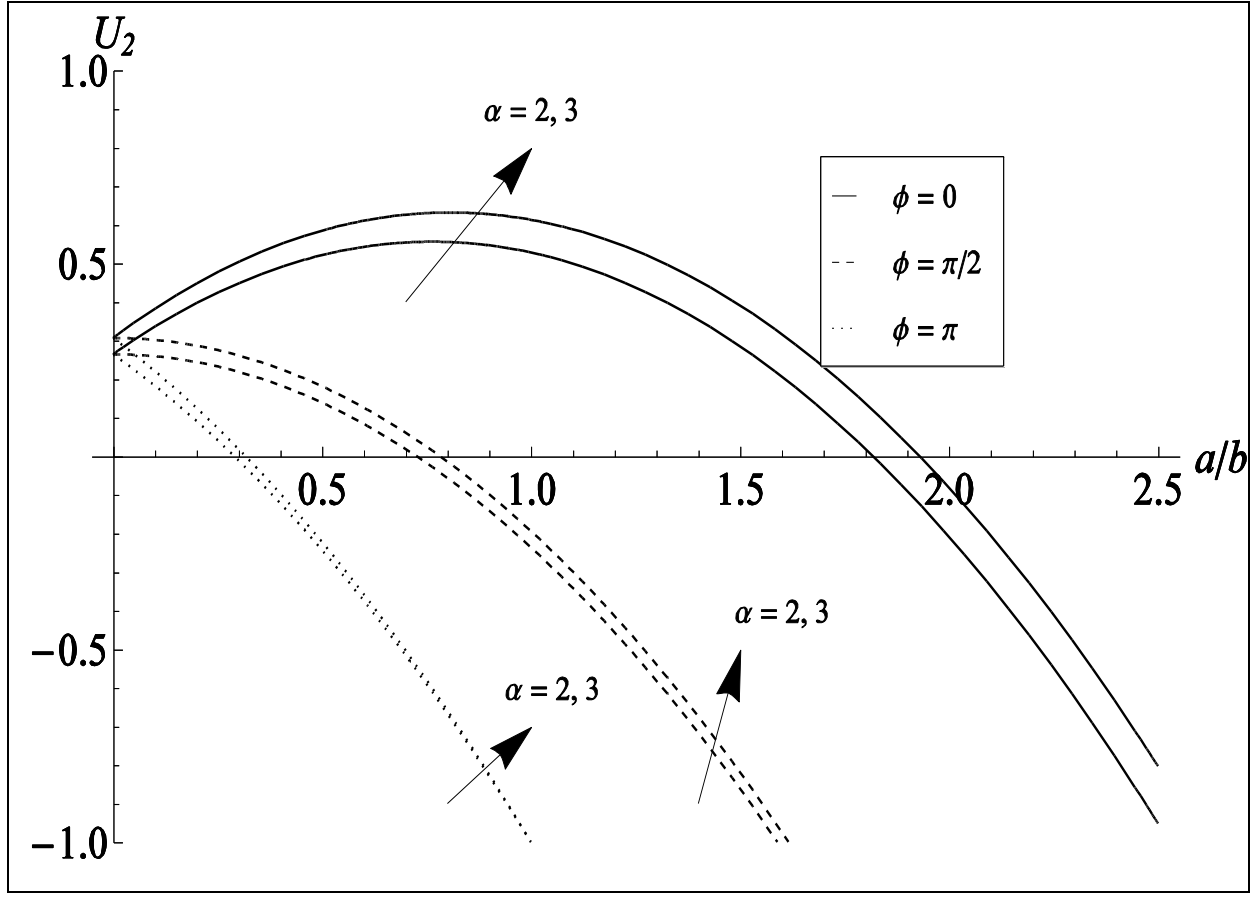

Fig. 5: Swimming speed as a function of $a / b$ for different values of $\phi$ and $\alpha$. 


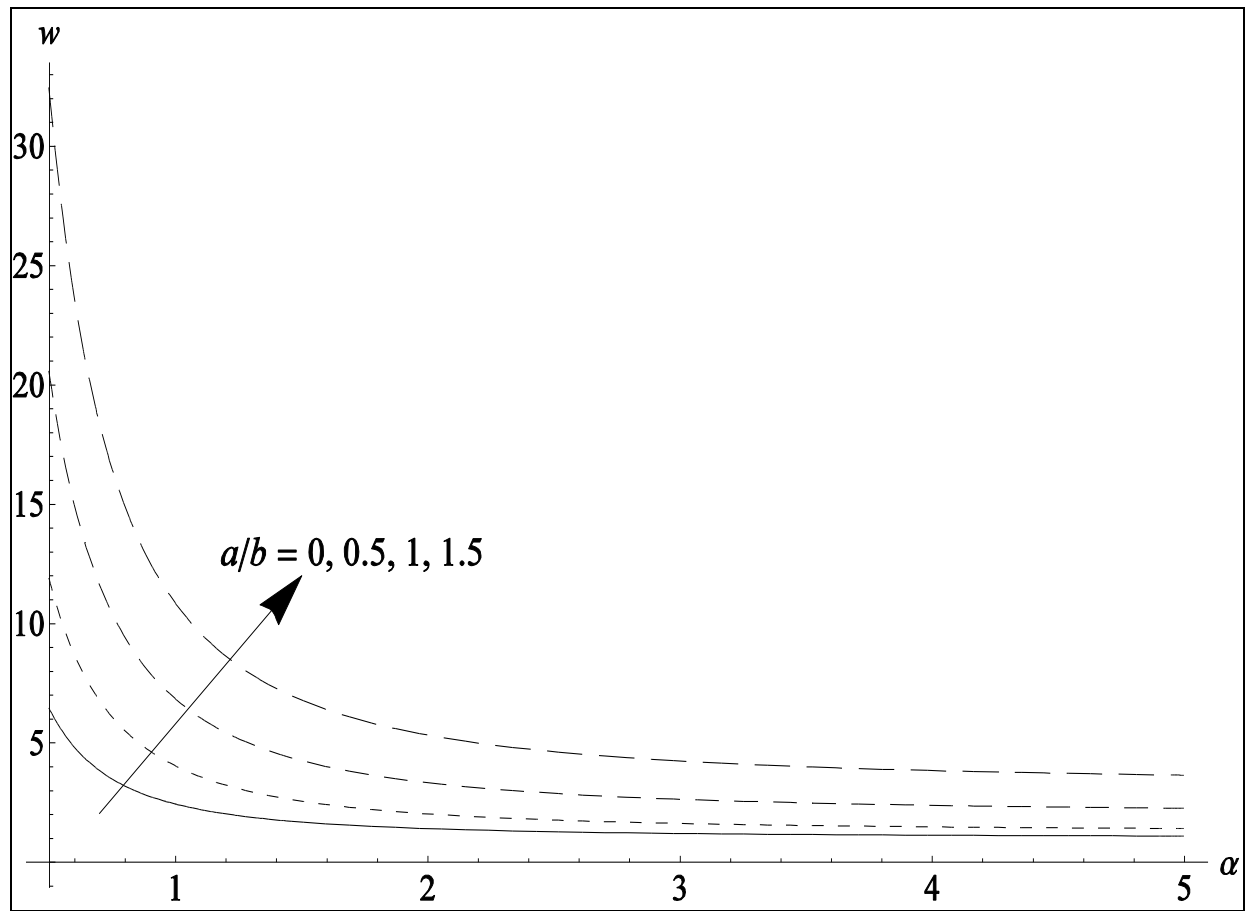

Fig. 6: Rate of work done by the sheet as a function of $\alpha$ for different values of $a / b$. 


\section{TABLES}

Table 1: Comparison of stream function obtained via perturbation and numerical methods for $a / b=1$,

$$
\phi=\pi / 4, x=0 \text { and } \varepsilon=0.1 \text {. }
$$

\begin{tabular}{|l|l|l|l|l|l|l|l|l|}
\hline \multirow{3}{*}{$y$} & \multicolumn{9}{|c|}{$\psi$} \\
\cline { 2 - 9 } & \multicolumn{2}{|c|}{$\alpha=1$} & $\alpha=5$ & \multicolumn{2}{l|}{$\alpha=10$} & $\alpha=100$ \\
\cline { 2 - 9 } & $\begin{array}{l}\text { Perturbation } \\
\text { solution }\end{array}$ & NTS & $\begin{array}{l}\text { Perturbation } \\
\text { solution }\end{array}$ & NTS & $\begin{array}{l}\text { Perturbation } \\
\text { solution }\end{array}$ & NTS & $\begin{array}{l}\text { Perturbatio } \\
\text { n solution }\end{array}$ & NTS \\
\hline 0 & 0 & 0 & 0 & 0 & 0 & 0 & 0 & 0 \\
\hline 1 & -0.032184 & -0.032180 & -0.024432 & -0.024420 & -0.0224566 & -0.0224569 & -0.020748 & -0.020749 \\
\hline 2 & -0.024230 & -0.024227 & -0.011450 & -0.011452 & -0.00861953 & -0.00861950 & -0.005778 & -0.005775 \\
\hline 4 & -0.000673 & -0.000669 & 0.013132 & 0.013129 & 0.0173278 & 0.0173280 & 0.022417 & 0.022419 \\
\hline 6 & 0.010435 & 0.010431 & 0.026876 & 0.026878 & 0.0328552 & 0.0328555 & 0.04037 & 0.04039 \\
\hline 8 & 0.016188 & 0.016183 & 0.037128 & 0.037129 & 0.0450417 & 0.0450419 & 0.055039 & 0.055041 \\
\hline 10 & 0.020644 & 0.020647 & 0.046629 & 0.046631 & 0.056511 & 0.0565114 & 0.069005 & 0.069007 \\
\hline
\end{tabular}

Table 2: Comparison of swimming speed obtained via perturbation and numerical methods for different values of $\alpha(a / b=0.5, \phi=0)$ and $a / b(\alpha=5, \phi=\pi / 4)$.

\begin{tabular}{|l|l|l|l|l|l|}
\hline \multirow{2}{*}{$\alpha$} & \multicolumn{2}{|c|}{$U$} & $a / b$ & \multicolumn{2}{c|}{$U$} \\
\cline { 2 - 3 } \cline { 5 - 6 } & $\begin{array}{l}\text { Perturbation } \\
\text { solution }\end{array}$ & NTS & & $\begin{array}{l}\text { Perturbation } \\
\text { solution }\end{array}$ & NTS \\
\hline 1 & 0.43566 & 0.435662 & 0.1 & 0.414886 & 0.414888 \\
\hline 2 & 0.520898 & 0.520899 & 0.5 & 0.537886 & 0.537883 \\
\hline 5 & 0.663703 & 0.663705 & 1 & 0.466636 & 0.466639 \\
\hline 10 & 0.750517 & 0.750520 & 1.2 & 0.368136 & 0.368140 \\
\hline 100 & 0.860295 & 0.860297 & 1.5 & 0.145386 & 0.145387 \\
\hline
\end{tabular}

